\title{
Defect Tolerance to Intolerance in the Vacancy-Ordered Double Perovskite Semiconductors $\mathrm{Cs}_{2} \mathrm{SnI}_{6}$ and $\mathrm{Cs}_{2} \mathrm{Tel}_{6}$
}

Annalise E. Maughan, ${ }^{\dagger}$ Alex M. Ganose, ${ }^{\ddagger}, \S$ Mitchell M. Bordelon, ${ }^{\dagger}$ Elisa M. Miller, ${ }^{\Perp}$ David O. Scanlon, ${ }^{\ddagger}, \S$ and James R. Neilson* ${ }^{\dagger}$

\author{
${ }^{\dagger}$ Department of Chemistry, Colorado State University, Fort Collins, Colorado 80523-1872, United States \\ ${ }^{\ddagger}$ University College London, Kathleen Lonsdale Materials Chemistry, Department of Chemistry, 20 Gordon Street, London WC1H \\ 0AJ, United Kingdom \\ ${ }^{\S}$ Diamond Light Source, Ltd., Diamond House, Harwell Science and Innovation Campus, Didcot, Oxfordshire OX11 0DE, United \\ Kingdom \\ "Chemical and Materials Sciences Center, National Renewable Energy Laboratory, 15013 Denver West Parkway, Golden, Colorado \\ 80401, United States
}

\section{Supporting Information}

ABSTRACT: Vacancy-ordered double perovskites of the general formula $A_{2} B X_{6}$ are a family of perovskite derivatives composed of a face-centered lattice of nearly isolated $\left[B X_{6}\right]$ units with $A$-site cations occupying the cuboctahedral voids. Despite the presence of isolated octahedral units, the closepacked iodide lattice provides significant electronic dispersion, such that $\mathrm{Cs}_{2} \mathrm{SnI}_{6}$ has recently been explored for applications in photovoltaic devices. To elucidate the structure-property relationships of these materials, we have synthesized solidsolution $\mathrm{Cs}_{2} \mathrm{Sn}_{1-x} \mathrm{Te}_{x} \mathrm{I}_{6}$. However, even though tellurium substitution increases electronic dispersion via closer I-I contact distances, the substitution experimentally yields insulating behavior from a significant decrease in carrier concentration and mobility. Density functional calculations of native defects in $\mathrm{Cs}_{2} \mathrm{SnI}_{6}$ reveal that iodine vacancies exhibit a low enthalpy of formation, and that the defect energy level is a shallow donor to the conduction band rendering the material tolerant to these defect states. The increased covalency of Te-I bonding renders the formation of iodine vacancy states unfavorable and is responsible for the reduction in conductivity upon Te substitution. Additionally, $\mathrm{Cs}_{2} \mathrm{TeI}_{6}$ is intolerant to the formation of these defects, because the defect level occurs deep within the band gap and thus localizes potential mobile charge carriers. In these vacancy-ordered double perovskites, the close-packed lattice of iodine provides significant electronic dispersion, while the interaction of the $B$ - and $X$-site ions dictates the properties as they pertain to electronic structure and defect tolerance. This simplified perspective based on extensive experimental and theoretical analysis provides a platform from which to understand structure-property relationships in functional perovskite halides

\section{INTRODUCTION}

Hybrid inorganic/organic and all-inorganic halide-based perovskites, such as $\left(\mathrm{CH}_{3} \mathrm{NH}_{3}\right) \mathrm{PbI}_{3}$ and $\mathrm{CsSnI}_{3}$, have been recently shown to exhibit advantageous optical and electronic properties for applications in field-effect transistors, ${ }^{1,2}$ light-emitting diodes, ${ }^{3,4}$ and efficient photovoltaic devices. ${ }^{5-9}$ However, these materials challenge conventional wisdom in that they exhibit excellent electrical performance despite the inexpensive, low-temperature, solution-based preparatory routes that result in imperfect materials with small grain sizes. ${ }^{10-14}$ Many of these perovskite halides suffer from several issues that currently preclude their use in widespread commercial applications, including lead toxicity, ${ }^{15,16}$ air and moisture sensitivity, ${ }^{17-22}$ and crystalline phase stability. ${ }^{6,23-26}$

The immense compositional and structural diversity of the perovskite family provides many opportunities to circumvent the challenges that accompany the $A \operatorname{Sn} X_{3}$ and $A \mathrm{~Pb} X_{3}$ perovskite halides. As in the perovskite oxides, ${ }^{27}$ the halides also undergo symmetry-lowering phase transitions upon cooling, manifesting as cooperative tilting of the $\mathrm{BI}_{6}$ octahedra, which influences the band gap and conductivity. ${ }^{6,28-31}$ The halides can also form ordered double perovskites of the general formula $A_{2} B^{\prime} B^{\prime \prime} X_{6}$, in which there is rock-salt ordering of the $B^{\prime}$ and $B^{\prime \prime}$ metals; the ordering is often driven by a large charge difference between the two metals. ${ }^{32}$ The recently reported double perovskite halides, $\mathrm{Cs}_{2} \mathrm{AgBiX}_{6}\left(X=\mathrm{Cl}^{-}\right.$and $\left.\mathrm{Br}^{-}\right),{ }^{33,34}$ absorb visible light with long carrier recombination lifetimes, providing new opportunities for nontoxic and air-stable alternatives to $\mathrm{Pb}^{2+}$ - and $\mathrm{Sn}^{2+}$-based $A B X_{3}$ perovskites.

Replacing one $B$-site cation with a vacancy in the $A_{2} B^{\prime} B^{\prime \prime} X_{6}$ double perovskite yields the vacancy-ordered double perov-

Received: March 28, 2016

Published: June 10, 2016 
skites, $A_{2} B X_{6}$ (e.g., $A_{2} B \square X_{6}$, where $\square$ is a vacancy), as depicted in Figure 1. Often referred to as antifluorite materials, these

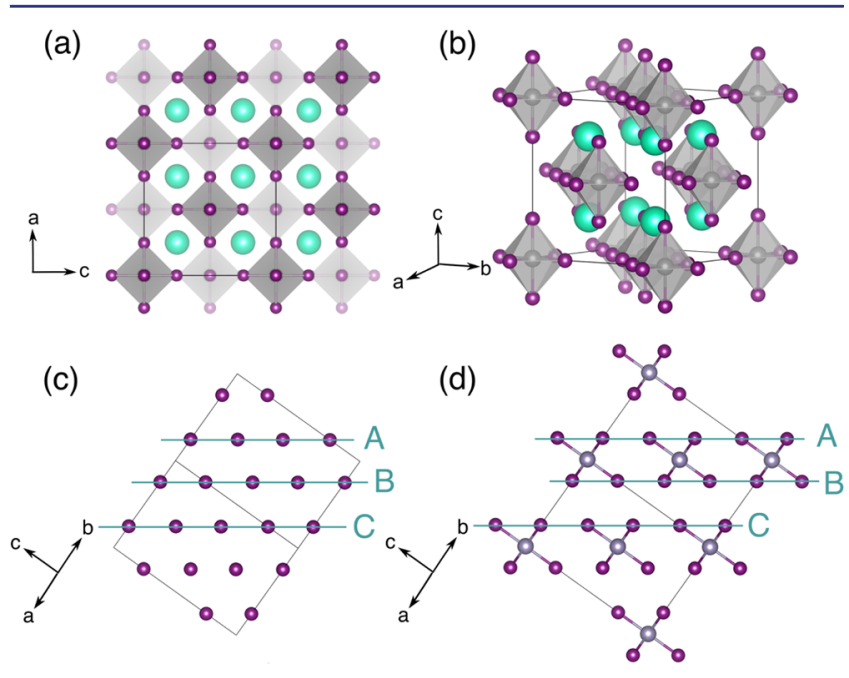

Figure 1. (a) Crystal structure of the vacancy-ordered double perovskites, $\mathrm{Cs}_{2} \mathrm{SnI}_{6}$ and $\mathrm{Cs}_{2} \mathrm{TeI}_{6}$. (b) Reorientation of the unit cell reveals the isolated octahedral units. Panel c illustrates the cubic closepacked iodine sub lattice, and panel $\mathrm{d}$ shows how the cubic packed iodine sub lattice is decorated with $\mathrm{Sn}$ atoms to yield the isolated octahedral units. Teal spheres are cesium, gray spheres are tin/ tellurium, and purple spheres are iodine.

defect-variant perovskites adopt the $\mathrm{K}_{2} \mathrm{PtCl}_{6}$ structure type, which can be described as isolated $\left[B X_{6}\right]^{2-}$ octahedra bridged by $A^{+}$cations. These $A_{2} B X_{6}$ perovskites exhibit several similarities to $A B X_{3}$ perovskites. Although every other $\left[B X_{6}\right]$ octahedron is removed, the close-packed anionic lattice familiar to $A B X_{3}$ perovskites is retained. Additionally, the vacancy-ordered structure type undergoes cooperative octahedral tilting and rotations in symmetry-lowering phase transitions upon cooling. ${ }^{35-38}$ As in $A B X_{3}$ perovskites, these phase transitions are driven by a mismatch in ionic radii of the constituent atoms: In $A B X_{3}$ perovskites, structural stability is predicted by the Goldschmidt tolerance factor, ${ }^{39,40}$ whereas the "radius ratio" of $A$-site cation radius to the radius of the 12-coordinate void has been used in the $A_{2} B X_{6}$ perovskites. $^{41}$

$\mathrm{Cs}_{2} \mathrm{SnI}_{6}$ adopts the cubic structure at room temperature $(\mathrm{F} m \overline{3} \mathrm{~m})$, as predicted by its radius ratio $\sim 0.94,{ }^{41}$ and the compound exhibits intrinsic n-type electrical conductivity, air and moisture stability, and strong visible light absorption, all of which are advantageous properties for photovoltaic devices. ${ }^{42,43}$ Despite the presence of regularly ordered $B$-site vacancies, the room-temperature carrier mobility of $\mathrm{Cs}_{2} \mathrm{SnI}_{6}$ is reported to be of the same order of magnitude as that of $\mathrm{CsSnI}_{3}(\mu \approx 310$ and $585 \mathrm{~cm}^{2} \mathrm{~V}^{-1} \mathrm{~s}^{-1}$, respectively); ${ }^{6,42}$ however, a more recent report on $\mathrm{Cs}_{2} \mathrm{SnI}_{6}$ thin films has determined a moderate electron mobility $\left(\mu_{\mathrm{e}} \approx 3 \mathrm{~cm}^{2} \mathrm{~V}^{-1} \mathrm{~s}^{-1}\right){ }^{43}$ The origin of conductivity in $\mathrm{Cs}_{2} \mathrm{SnI}_{6}$ has been hypothesized to stem from (a) the presence of dispersive conduction band states and (b) the formation of iodine vacancies, which serve as electron donors that contribute to conductivity. ${ }^{42-44}$ The seemingly inherent "defect-tolerance" of $\mathrm{Cs}_{2} \mathrm{SnI}_{6}$ motivates exploration of these molecular perovskites and the effect of composition on their tolerance to crystallographic defects. The concept of defect tolerance has been employed to identify materials that are insensitive to charge localization due to intrinsic defects ${ }^{45}$ and has previously been applied to $A B X_{3}$ perovskites. ${ }^{46,47}$
In this contribution, we describe the structure-property relationships of the solid solution, $\mathrm{Cs}_{2} \mathrm{Sn}_{1-x} \mathrm{Te}_{x} \mathrm{I}_{6}$, which characterizes $\mathrm{Cs}_{2} \mathrm{SnI}_{6}$ as a defect-tolerant semiconductor, thus illustrating how the close-packed anionic lattice of the vacancyordered perovskite halides can yield advantageous electronic properties, despite the lack of $B-X-B$ connectivity. In these materials, the tin(IV) ion (formal $[\mathrm{Kr}] 4 \mathrm{~d}^{10} 5 \mathrm{~s}^{0}$ electron configuration) is substituted with the larger tellurium(IV) ion (formal $[\mathrm{Kr}] 5 \mathrm{~d}^{10} 5 \mathrm{~s}^{2}$ electron configuration) to yield a solid solution with no distortions of the local coordination environment, despite the presence of the tellurium lone pair. In this work, we refer exclusively to formal oxidation state of the $\mathrm{Sn}$ and $\mathrm{Te}$ ions in these materials (i.e., $\mathrm{Sn}^{4+}$ and $\mathrm{Te}^{4+}$ ), because the actual charge is likely reduced through covalent bonding interactions. ${ }^{48,49}$ The larger $\mathrm{Te}(\mathrm{IV})$ ion is accommodated into the structure by expansion of the $\left[\mathrm{BI}_{6}\right]$ octahedra at the expense of the inter-octahedral void region. Despite the reduced I-I separation between neighboring octahedra and higher predicted carrier mobilities, substitution of tellurium is accompanied by a significant reduction in electrical conductivity from a dramatically reduced carrier concentration and carrier mobility. There is a nonlinear change in the optical gap with increasing $\mathrm{Te}$ substitution, yet no distortions of the crystal structure or local coordination environment are observed across the solid solution, as determined by analysis of high-resolution time-of-flight neutron and synchrotron X-ray diffraction data and X-ray pair distribution function analysis. From density functional calculations, we have identified iodine vacancies as the lowest energy defect in $\mathrm{Cs}_{2} \mathrm{SnI}_{6}$, which act as shallow electron donors. In contrast, iodine vacancies in $\mathrm{Cs}_{2} \mathrm{TeI}_{6}$ have a higher enthalpy of formation and form deep in the band gap, a consequence of increased Te-I covalency, which renders $\mathrm{Cs}_{2} \mathrm{TeI}_{6}$ defect intolerant. While the close-packed halogen sublattice provides a framework for mobile charge carriers in these vacancy-ordered double perovskites, the interaction between the $B$-site cation and $X$-site anions serves to set the magnitude and character of the band gap and dictates the tolerance of these materials to point defects in the lattice.

\section{RESULTS AND DISCUSSION}

Electronic Properties. $\mathrm{Cs}_{2} \mathrm{SnI}_{6}$ exhibits properties consistent with an intrinsically doped $n$-type semiconductor. As measured on a cold-pressed polycrystalline pellet, the resistivity of $\mathrm{Cs}_{2} \mathrm{SnI}_{6}$ at $T=300 \mathrm{~K}$ is $\rho \approx 13 \Omega \cdot \mathrm{cm}$. The exponential increase in resistivity on cooling is consistent with semiconducting behavior (Figure 2a), and the data follow $\rho=\rho_{0} \exp \left[\frac{T_{0}}{T}\right]^{p}$, where $p=0.56$, consistent with EfrosShklovskii variable range hopping (ES-VRH) ${ }^{50,51}$ However, the precise nature of the transport is yet inconclusive due to the polycrystalline nature of the specimens. Measurement of the Hall voltage at $T=300 \mathrm{~K}$ confirms intrinsic $n$-type behavior, with a carrier concentration of $n_{\mathrm{e}} \approx 5(1) \times 10^{16}$ and a carrier mobility of $\mu_{\mathrm{e}} \approx 8.6(5) \mathrm{cm}^{2} \mathrm{~V}^{-1} \mathrm{~s}^{-1}$ (Figure $2 \mathrm{c}$ ).

Partial substitution of $\mathrm{Sn}$ by Te in the solid-solution series $\mathrm{Cs}_{2} \mathrm{Sn}_{1-x} \mathrm{Te}_{x} \mathrm{I}_{6}$ increases the electrical resistivity of the materials. At $5 \%$ tellurium substitution $(x=0.05)$, a 50 -fold increase in resistivity is observed, and samples containing more than $25 \%$ $\mathrm{Te}$ are too insulating for accurate measurements (Figure $2 \mathrm{~b}$ ). Measurements of the Hall voltage for the 5 and $10 \% \mathrm{Te}$ samples reveal that while the dominant carriers are still electrons, the carrier concentrations and mobility are dramatically reduced (Figure $2 \mathrm{c}$ ). Therefore, detailed analyses 

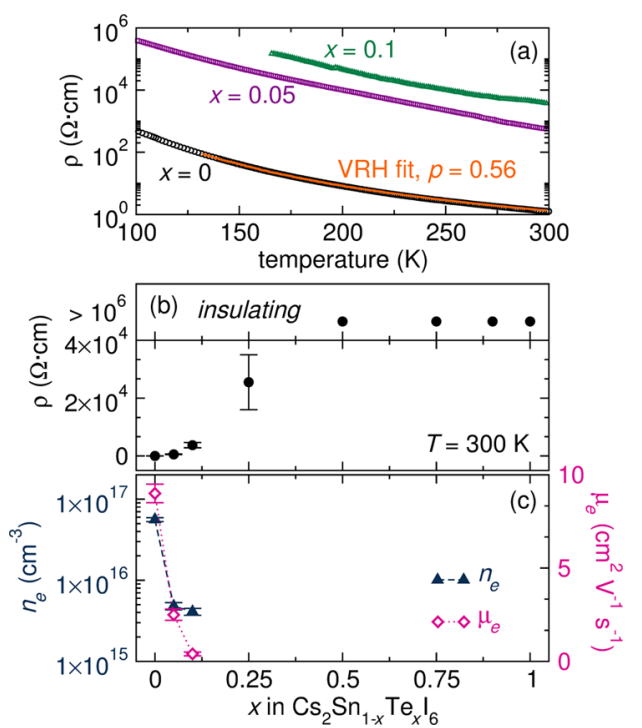

Figure 2. (a) Temperature dependence of the electrical resistivity for the solid solution $\mathrm{Cs}_{2} \mathrm{Sn}_{1-x} \mathrm{Te}_{x} \mathrm{I}_{6}$. The data for $\mathrm{Cs}_{2} \mathrm{SnI}_{6}$ (black circles) indicate semiconducting behavior, as described by variable rangehopping conductivity from $T=130-300 \mathrm{~K}$ (orange line). Temperature-dependent curves are also shown for $x=0.05$ (purple squares) and $x=0.1$ (green triangles). (b) Room-temperature resistivity for $x=$ $0,0.05,0.1$, and 0.25 in $\mathrm{Cs}_{2} \mathrm{Sn}_{1-x} \mathrm{Te}_{x} \mathrm{I}_{6}$. The $x=0.5,0.75,0.9$, and 1 samples were too resistive for accurate measurements. (c) Carrier concentration, $n_{\mathrm{e}}$ (filled teal triangles), and carrier mobility, $\mu_{\mathrm{e}}$ (open pink diamonds), for $x=0,0.05$, and 0.1 are reduced upon Te substitution, as determined by Hall voltage measurements. Error bars denote the propagation of uncertainty from the sample dimensions for all samples, except for $x=0.05$, which denotes the standard deviation of 3 transport specimens.

of the crystal and electronic structures are necessary to determine the nature of resistivity increase.

Crystal Structures. High-resolution time-of-flight neutron powder diffraction data for $\mathrm{Cs}_{2} \mathrm{SnI}_{6}$ and $\mathrm{Cs}_{2} \mathrm{TeI}_{6}$ collected at $T=300$ and $10 \mathrm{~K}$ reveal cubic crystal structures. Structural parameters and refinement statistics can be found in Table 1 . The diffraction patterns are all consistent with the cubic, vacancy-ordered double perovskite structure $(F m \overline{3} m$, Figures 1 and 3). Rietveld analysis yields lattice parameters of 11.65272(4) $\AA$ and 11.7088(1) $\AA$ for $\mathrm{Cs}_{2} \mathrm{SnI}_{6}$ and $\mathrm{Cs}_{2} \mathrm{TeI}_{6}$, respectively, at $T=300 \mathrm{~K}$. The lack of sharp Bragg features beyond $Q>8 \AA^{-1}$ at $T=300 \mathrm{~K}$ suggests the presence of significant rotational disorder of the $\mathrm{BI}_{6}$ octahedral units, which is not uncommon in crystalline materials containing isolated polyhedra $^{52,53}$ and is reflected in the somewhat large I anisotropic displacement parameters $\left(U_{22}\right.$ and $\left.U_{33}\right)$ generated during the refinement (Table 1). The radius ratios of $\mathrm{Cs}_{2} \mathrm{SnI}_{6}$ $\left(r_{\mathrm{A}} / r_{\mathrm{H}}=0.94\right)$ and $\mathrm{Cs}_{2} \mathrm{TeI}_{6}\left(r_{\mathrm{A}} / r_{\mathrm{H}}=0.97\right)$ fall in the range of $0.89<r<1$; thus, these materials are not expected to undergo symmetry-lowering phase transitions upon cooling, consistent with neutron-scattering data collected at $T=10 \mathrm{~K}^{41,54-56}$

The crystallography is further supported by the absence of any entropy-releasing anomalies in the experimentally measured low-temperature heat capacity. Instead, the specific heat reveals the presence of low-energy localized lattice vibrations, as described by the summation of the Einstein and Debye models of specific heat (Figure S1). Analysis of the data according to these models yields Debye temperatures of $\Theta_{D}=149(2)$ and $122(2) \mathrm{K}$ and localized (Einstein) oscillator energies of $\epsilon=$ 3.58(4) and 3.27(4) $\mathrm{meV}$ for $\mathrm{Cs}_{2} \mathrm{SnI}_{6}$ and $\mathrm{Cs}_{2} \mathrm{TeI}_{6}$, respectively,
Table 1. Structural Parameters and Refinement Statistics for $\mathrm{Cs}_{2} \mathrm{SnI}_{6}$ and $\mathrm{Cs}_{2} \mathrm{TeI}_{6}$ from High-Resolution Neutron Diffraction Data at $T=300$ and $10 \mathrm{~K}^{a}$

\begin{tabular}{lcl} 
& \multicolumn{1}{c}{$\mathrm{Cs}_{2} \mathrm{SnI}_{6}$} & $\mathrm{Cs}_{2} \mathrm{TeI}_{6}$ \\
$a(\AA)$ & $T=0 \mathrm{~K}$ & \\
$\mathrm{I}(x, 0,0), 24 \mathrm{e}$ & $11.65272(4)$ & $11.7088(1)$ \\
$U_{\text {iso }}(\mathrm{Cs})\left(\AA^{2}\right)$ & $0.2452(1)$ & $0.2498(2)$ \\
$U_{\text {iso }}(B)\left(\AA^{2}\right)$ & $0.0393(4)$ & $0.0445(7)$ \\
$U_{11}(\mathrm{I})\left(\AA^{2}\right)$ & $0.0159(4)$ & $0.0262(7)$ \\
$U_{22}=U_{33}(\mathrm{I})\left(\AA^{2}\right)$ & $0.0104(3)$ & $0.0142(6)$ \\
red. $\chi^{2}$ & $0.0396(2)$ & $0.0444(4)$ \\
$w R$ & 2.55 & 1.958 \\
& $3.27 \%$ & $3.88 \%$ \\
$a(\AA)$ & $T=10 \mathrm{~K}$ & \\
$\mathrm{I}(x, 0,0), 24 \mathrm{e}$ & $11.53346(3)$ & $11.5895(1)$ \\
$U_{\text {iss }}(\mathrm{Cs})\left(\AA^{2}\right)$ & $0.2481(1)$ & $0.2514(2)$ \\
$U_{\text {iso }}(B)\left(\AA^{2}\right)$ & $0.00435(9)$ & $0.0064(2)$ \\
$U_{11}(\mathrm{I})\left(\AA^{2}\right)$ & $0.0054(1)$ & $0.0114(4)$ \\
$U_{22}=U_{33}(\mathrm{I})\left(\AA^{2}\right)$ & $0.00308(3)$ & $0.00497(7)$ \\
red. $\chi^{2}$ & $0.00277(8)$ & $0.0052(1)$ \\
$w R$ & 4.498 & 4.399 \\
$w R$ & $4.43 \%$ & $6.03 \%$
\end{tabular}

${ }^{a}$ Space group: $F m \overline{3} m$. Sn/Te position, 4a: $(0,0,0)$; Cs position, $8 \mathrm{c}$ : $(0.25,0.25,0.25)$.

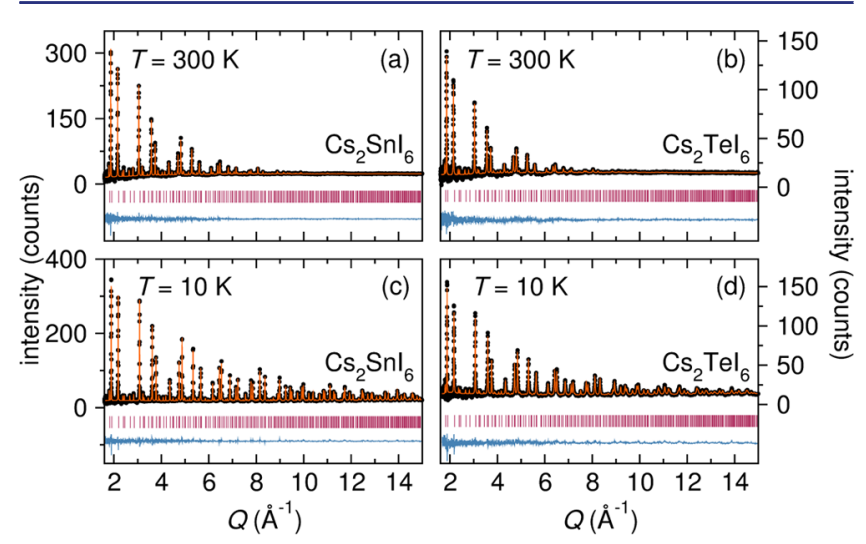

Figure 3. Rietveld refinements of high-resolution time-of-flight neutron scattering data of (a and c) $\mathrm{Cs}_{2} \mathrm{SnI}_{6}$ and (b and d) $\mathrm{Cs}_{2} \mathrm{TeI}_{6}$ collected from wavelength frame 2 on the POWGEN diffractometer at the Spallation Neutron Source, Oak Ridge National Laboratory. Data collected at $T=300 \mathrm{~K}$ are shown in panels a and $\mathrm{b}$, and data collected at $T=10 \mathrm{~K}$ are shown in panels $\mathrm{c}$ and $\mathrm{d}$. Refinements were performed using data collected from wavelength frames 2 and 4 of the POWGEN diffractometer (data from frame 4 are shown in Figure S2.) Black circles are the data, the orange line is the fit, and the blue line is the difference. The pink tick marks indicate the location of predicted Bragg reflections for the cubic structure.

and is consistent with the presence of localized octahedral rotations. $^{57}$

High-resolution synchrotron powder X-ray diffraction (SXRD) data collected for all members in the $\mathrm{Cs}_{2} \mathrm{Sn}_{1-x} \mathrm{Te}_{x} \mathrm{I}_{6}$ series at room temperature confirm solid-solution behavior. SXRD data for the entire solid solution present reflections consistent with the cubic vacancy-ordered double perovskite structure (Figure 4a), and the noticeable shift in the reflection positions to lower $Q$ with increasing $x$ is consistent with an expansion of the unit cell upon substitution of the larger tellurium ion, as shown in Figure $4 \mathrm{~b}$. Structural models for the solid solution were constructed by modifying the $\mathrm{Sn} / \mathrm{Te}$ 

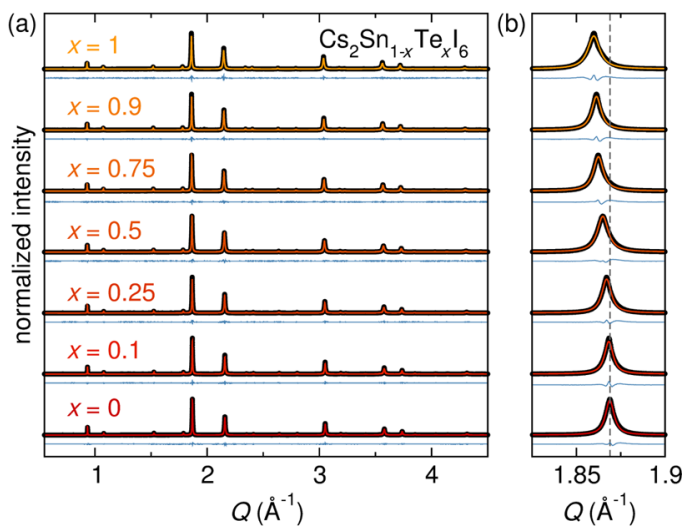

Figure 4. (a) High-resolution synchrotron X-ray diffraction data (11$\mathrm{BM}$ ) and Rietveld refinements of the solid-solution series $\mathrm{Cs}_{2} \mathrm{Sn}_{1-x} \mathrm{Te}_{x} \mathrm{I}_{6}$ collected at room temperature (black circles represent data, colored lines represent the fit, and blue lines are difference curves). (b) The strongest reflection, $\{222\}$ at $Q=1.87 \AA^{-1}$, shifts to lower $Q$ with increasing tellurium substitution.

occupancy to reflect the nominal stoichiometry utilized during the syntheses. The resulting Rietveld refinements are shown in Figure 4, and the structural parameters and refinement statistics are compiled in Table 2.

The refined lattice parameters follow a linear increase upon tellurium substitution, as shown in Figure 5a. While the increase in unit cell volume is not altogether unsurprising given the increase in B-site ionic radius from $r_{\mathrm{Sn}}=0.69 \AA$ to $r_{\mathrm{Te}}=0.97$ $\AA{ }^{55}$ the $0.5 \%$ increase in unit cell parameter is insufficient to account for the $40.5 \%$ increase in ionic radius from $\mathrm{Sn}^{4+}$ to $\mathrm{Te}^{4+}$. From the crystallographic analysis of the structures with only one free internal structural coordinate, the iodine position $(x, 0,0)$, the larger $\mathrm{Te}^{4+}$ ion is accommodated into the structure with an increase of the average $B-I$ bond length by $1.6 \%$ at the expense of the I-I contact distances between octahedra (interoctahedral), as shown in Figure 5c.

To rule out the presence of distortions in the local coordination environment or stereochemical lone-pair activity of the $\mathrm{Te}(\mathrm{IV}) 5 \mathrm{~s}^{2}$ electrons, the X-ray pair distribution function (XPDF) for each compound in the solid solution was computed from synchrotron X-ray total scattering data (beamline 11-ID-B). As shown in Figure 6, the XPDFs are nearly identical across the solid solution, with the exception of the increasing coordination distance of the nearest neighbor $(B-I)$ and decreasing coordination distance of the next-nearest neighbor (I-I) pair correlations, as highlighted in the left panel of Figure 6. Interestingly, we have found that the PDFs of the intermediate members of the solid solution can be modeled as a

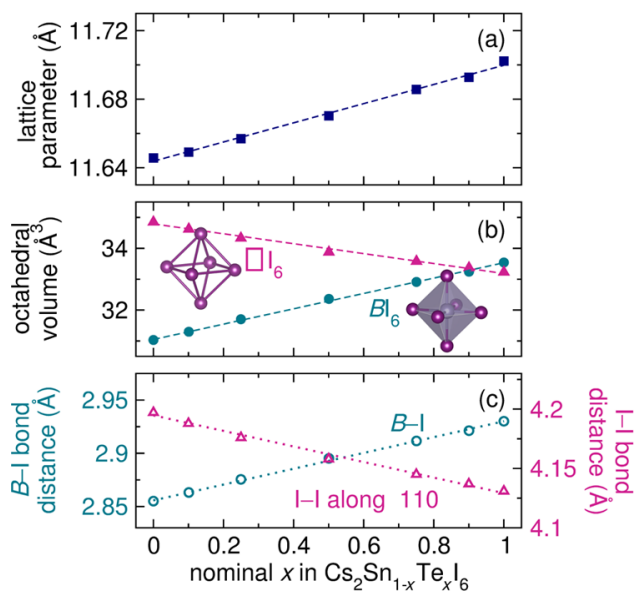

Figure 5. Crystal structure parameters across the solid-solution series, $\mathrm{Cs}_{2} \mathrm{Sn}_{1-x} \mathrm{Te}_{x} \mathrm{I}_{6}$, as determined from high-resolution synchrotron X-ray diffraction at room temperature. (a) The refined lattice parameters are plotted as a function of nominal $x$ in $\mathrm{Cs}_{2} \mathrm{Sn}_{1-x} \mathrm{Te}_{x} \mathrm{I}_{6}$. The dashed line represents Vegard's law interpolated from the end members. (b) The average polyhedral volumes of the $B \mathrm{I}_{6}$ octahedra and $\square \mathrm{I}_{6}$ void regions show that the voids become smaller as the $\left[\mathrm{BI}_{6}\right]$ octahedra become larger. (c) The average $B-I$ bond distances and I-I contact distances along the $\langle 110\rangle$ directions in the unit cell reflect the trend shown in $b$. Dashed lines in $\mathrm{b}$ and $\mathrm{c}$ are linear regressions.

linear combination of the end member PDFs (i.e., XPDF$\left.\left(\mathrm{Cs}_{2} \mathrm{Sn}_{1-x} \mathrm{Te}_{x} \mathrm{I}_{6}\right)=(1-x) \mathrm{XPDF}_{\mathrm{Sn}}+(x) \mathrm{XPDF}_{\mathrm{Te}}\right)$, which indicates that the local structure of the solid solution is identical to that of the end members. This analysis is shown in Figure 6. The XPDFs extracted from the experimental data are shown as colored lines, and the computed linear combinations are overlaid as dotted lines. The differences between the data and the linear combination analyses are shown as blue lines. Full refinements of the XPDF data for each member of the solid solution are also included in Figure S3.

Density functional calculations of the LO-TO phonon splitting for $\mathrm{Cs}_{2} \mathrm{SnI}_{6}$ and $\mathrm{Cs}_{2} \mathrm{TeI}_{6}$ further support the experimental observation of a stereochemically inactive lone pair and are in good agreement with LO-TO splittings determined experimentally. ${ }^{58}$ As shown in the Supporting Information, the largest splitting across both compounds is 21 $\mathrm{cm}^{-1}$, which is similar in magnitude to other materials with stereochemically inactive lone-pairs such as PbTe $\left(52 \mathrm{~cm}^{-1}\right)^{59}$ and significantly smaller than in materials with distorted local geometries, such as $\mathrm{GeS}\left(322 \mathrm{~cm}^{-1}\right)^{60}$ and the cubic perovskite oxides (typically $\left.\sim 700 \mathrm{~cm}^{-1}\right) .{ }^{61}$ Analysis of the charge-density isosurface for the electron density range containing the $\mathrm{Sn}$ and

Table 2. Structural Parameters and Refinement Statistics for $\mathrm{Cs}_{2} \mathrm{Sn}_{1-x} \mathrm{Te}_{x} \mathrm{I}_{6}$ from Rietveld Refinements of High-Resolution Synchrotron Powder X-ray Diffraction Data

\begin{tabular}{|c|c|c|c|c|c|c|c|}
\hline$x$ & 0 & 0.1 & 0.25 & 0.5 & 0.75 & 0.9 & 1 \\
\hline stoichiometric $x$ & 0 & 0.101 & 0.2498 & 0.5007 & 0.7505 & 0.9003 & 1 \\
\hline$a(\AA)$ & $11.64572(2)$ & $11.64912(2)$ & $11.65697(2)$ & $11.67028(3)$ & $11.68567(3)$ & $11.69277(3)$ & $11.70220(4)$ \\
\hline $\mathrm{I}(x, 0,0)$ & $0.24516(4)$ & $0.24579(5)$ & $0.24668(5)$ & $0.24809(5)$ & $0.24916(5)$ & $0.24984(5)$ & $0.25039(6)$ \\
\hline$U_{\text {iso }}(\mathrm{Cs})\left(\AA^{2}\right)$ & $0.0402(2)$ & $0.0401(2)$ & $0.0413(2)$ & $0.0415(2)$ & $0.0434(3)$ & $0.0433(2)$ & $0.0447(3)$ \\
\hline$U_{\text {iso }}(B)\left(\AA^{2}\right)$ & $0.0276(3)$ & $0.0331(3)$ & $0.0308(3)$ & $0.0299(3)$ & $0.0290(3)$ & $0.0270(3)$ & $0.0302(4)$ \\
\hline$U_{11}(\mathrm{I})\left(\AA^{2}\right)$ & $0.0202(2)$ & $0.0190(2)$ & $0.0236(4)$ & $0.0237(2)$ & $0.0225(4)$ & $0.0201(3)$ & $0.0231(5)$ \\
\hline$U_{22}=U_{33}(\mathrm{I})\left(\AA^{2}\right)$ & $0.0444(1)$ & $0.0458(1)$ & $0.0450(2)$ & $0.0446(1)$ & $0.0470(2)$ & $0.0484(2)$ & $0.0489(3)$ \\
\hline Red. $\chi^{2}$ & 4.054 & 3.692 & 5.384 & 6.341 & 6.352 & 7.462 & 4.397 \\
\hline$w R$ & $7.49 \%$ & $8.60 \%$ & $7.99 \%$ & $8.06 \%$ & $8.04 \%$ & $8.85 \%$ & $8.60 \%$ \\
\hline
\end{tabular}




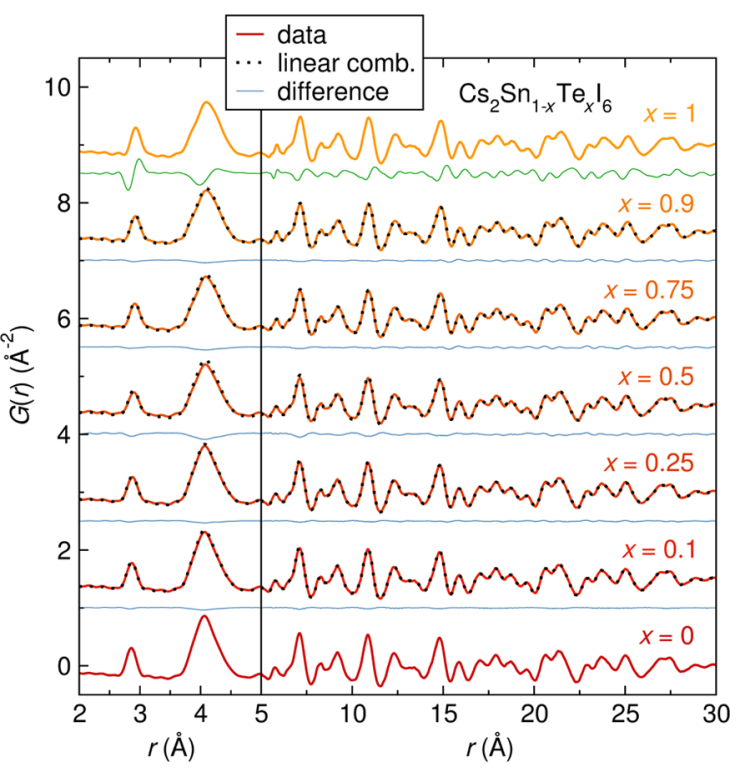

Figure 6. Synchrotron X-ray pair distribution function analysis of all compounds in the solid-solution series from data collected at room temperature. The XPDF data for all compounds can be constructed by a linear combination of the XPDF data for the end members (i.e., $\left.\mathrm{XPDF}=(1-x) \mathrm{XPDF}_{\mathrm{Sn}}+(x) \mathrm{XPDF}_{\mathrm{Te}}\right)$. The data are shown as solid colored lines, and the computed linear combinations for each member are overlain as a black dotted line. The difference between the data and the linear combinations are shown as blue lines. The difference curve shown below $x=1$ (green line) is the difference between endmembers and illustrates the apparent shift in bond lengths across the solid solution. Note the split $x$-axis to highlight the nearest-neighbor $(B-I)$ and next-nearest-neighbor (I-I) pair correlations.

Te $5 s^{2}$ electrons reveals no asymmetry in the electron density surrounding the metals (Figure S4). As such, it is clear that the compounds studied here do not display a tendency to structurally distort due to lone-pair stereochemical activity.

Optical Spectroscopy and Electronic States. UVvisible diffuse reflectance spectroscopy performed on powdered samples illustrates an increase in optical gap from $\mathrm{Cs}_{2} \mathrm{SnI}_{6}$ to $\mathrm{Cs}_{2} \mathrm{TeI}_{6}$ across the solid solution. While Tauc analysis is often used to extract the nature (direct vs indirect) and magnitude of the band gap from diffuse reflectance data, the method often underestimates the band gap of most crystalline semiconductors, particularly those that are degenerately doped or which exhibit delocalized electronic states. ${ }^{62}$ Therefore, the energies of the absorption onsets determined by UV-visible diffuse reflectance spectroscopy are used to approximate the optical gaps of these materials. The reflectance data were converted to absorbance, and the baseline and onset regions were fit to linear functions. The optical gap was determined by the intersection of the two fits, which yields the nonlinear relationship shown as the orange circles in Figure 7. In addition to the above method, we have also extracted values for optical gaps by several other methods (as elaborated upon in the Supporting Information), which give a range of values for the optical gap of each compound shown as blue bars in Figure 7 and highlight the ambiguity in determination of the optical gaps by diffuse reflectance spectroscopy.

Two previous studies of $\mathrm{Cs}_{2} \mathrm{SnI}_{6}$ report a band gap magnitude of $\sim 1.3 \mathrm{eV}$ but disagree regarding the assignment of direct versus indirect band gap, ${ }^{42,63}$ whereas another report suggests a direct band gap of $1.6 \mathrm{eV}$ for thin-film samples. ${ }^{43}$

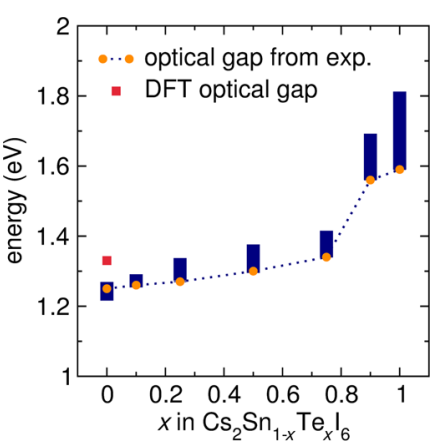

Figure 7. Optical gaps of the solid-solution series determined from UV-visible diffuse reflectance data. The optical gaps (shown as yellow circles) are determined by converting the reflectance data to absorbance, fitting linear functions to the baseline and onset regions of the data, and determining the intersection. The blue bars represent a range of values for optical gaps determined by extracting the data by various methods, which are discussed in more detail in the Supporting Information and are shown to highlight the ambiguity in determining the value of the optical gap by diffuse reflectance spectroscopy. The red square represents the magnitude of the DFT-calculated optical gap arising from the dipole-allowed transition below the valence band maximum (see Figure S8).

The analysis performed here suggests an optical gap of $\sim 1.25$ $\mathrm{eV}$ for $\mathrm{Cs}_{2} \mathrm{SnI}_{6}$. The same analysis for $\mathrm{Cs}_{2} \mathrm{TeI}_{6}$ gives an optical gap of $\sim 1.59 \mathrm{eV}$, which is in reasonable agreement with the previously reported indirect band gap of $1.5 \mathrm{eV} .{ }^{64}$ However, determination of the optical gap for $\mathrm{Cs}_{2} \mathrm{TeI}_{6}$ is complicated by the presence of exciton-like features near the absorption edge, which are also consistent with single-ion excited states arising from the $\mathrm{Te}(\mathrm{IV}) 5 \mathrm{~s}^{2}$ electron configuration. ${ }^{65-68}$ Photoluminescence (PL) measurements attempted on cold-pressed polycrystalline pellets of $\mathrm{Cs}_{2} \mathrm{SnI}_{6}$ and $\mathrm{Cs}_{2} \mathrm{TeI}_{6}$ did not show any measurable PL intensity, as shown in Figure S5. We note that $\mathrm{PL}$ was observed in thin-films of $\mathrm{Cs}_{2} \mathrm{SnI}_{6}{ }^{43}$ thus, we anticipate that the lack of signal is due to phonon quenching and differences arising from the bulk versus thin film nature of the specimens.

As shown in Figure 7, the optical gaps of these materials shift to higher energy with increased tellurium substitution. Between $x=0$ and 0.75 , the gaps increase steadily (and nearly linearly), and the spectra present well-defined onset regions. The optical gaps of the $x=0.9$ and 1 compounds do not follow the linear trend; however, determination of the optical gaps for the $x=$ 0.9 and 1 members of the solid solution is somewhat ambiguous, given the presence of peaks near the absorption edge. With that being said, the optical gaps monotonically increase across the solid solution and do not follow a parabolic band-bowing relationship [e.g., $E_{\mathrm{g}}=(1-x) E_{\mathrm{g}}^{\mathrm{Sn}}(x)+x E_{\mathrm{g}}^{\mathrm{Te}}(x)-$ $b x(x-1)]$, as often observed in solid-solutions. ${ }^{69-72}$

Density functional calculations of the electronic structures provide insight into the experimental observations. The calculations were performed using the Vienna $A b$ initio simulation package (VASP), ${ }^{73-76}$ with the HSE06 hybrid functional ${ }^{77}$ to provide an accurate description of the electronic structure; the calculations also included an explicit treatment of spin-orbit coupling (SOC) effects (illustrated in Figure S7). The band structure of $\mathrm{Cs}_{2} \mathrm{SnI}_{6}$ illustrates a direct band gap, $E_{\mathrm{g}}^{\text {dir }}$ $=0.97 \mathrm{eV}$, at the $\Gamma$-point, which is smaller than the experimentally observed optical gap of $E_{\mathrm{opt}}=1.25 \mathrm{eV}$. Calculation of the optical absorption reveals an onset that occurs at $1.33 \mathrm{eV}$, which is considerably larger than the direct 
band gap of $0.97 \mathrm{eV}$ (Figure 7, red square). The direct VB-CB optical transition at the $\Gamma$-point is dipole-forbidden (as elaborated upon in the Supporting Information, Figure S8). ${ }^{78-80}$ As such, prior reports may have employed artificially large amounts of Hartree-Fock (HF) exchange to fit the fundamental band gap to the experimental optical gap. ${ }^{43,44,81}$ In agreement with the HSE06+SOC calculations presented here, GWO calculations of $\mathrm{Cs}_{2} \mathrm{SnI}_{6}$ indicate a direct band gap of 0.88 $\mathrm{eV}^{58}$

The band structure of $\mathrm{Cs}_{2} \mathrm{TeI}_{6}$ indicates an indirect band gap with a magnitude $E_{\mathrm{g}}^{\text {ind }}=1.83 \mathrm{eV}$, which is larger than the experimentally observed optical gap of $1.59 \mathrm{eV}$. In $\mathrm{Cs}_{2} \mathrm{TeI}_{6}$, the highest occupied and lowest unoccupied bands appear at the $\mathrm{X}$ and $\mathrm{L}$ points in the first Brillouin zone, respectively, with the direct band gap $\left(E_{\mathrm{g}}^{\mathrm{dir}}=2.05 \mathrm{eV}\right)$ occurring at L. The discrepancy between the DFT-calculated band gap and the experimentally determined optical gap may arise from the fact that VASP calculations do not take into account absorption arising from configuration interactions with different excited states, ${ }^{65-68}$ which may obscure the absorption edge.

Analysis of the orbital character of $\mathrm{Cs}_{2} \mathrm{SnI}_{6}$ and $\mathrm{Cs}_{2} \mathrm{TeI}_{6}$ reveals that the highest occupied bands in both compounds are comprised of I 5 p character (Figure 8). For $\mathrm{Cs}_{2} \mathrm{SnI}_{6}$, the states at the highest occupied band are primarily nonbonding, but for $\mathrm{Cs}_{2} \mathrm{TeI}_{6}$, these are antibonding in character as determined by crystal orbital Hamilton population (COHP) analysis of the $B-$ I interactions (Figure S9). ${ }^{82}$ Similar to previous hybrid DFT studies on $\mathrm{Cs}_{2} \mathrm{SnI}_{6}$, ${ }^{44,81}$ there are occupied $\mathrm{Sn} 5$ s states around (a)

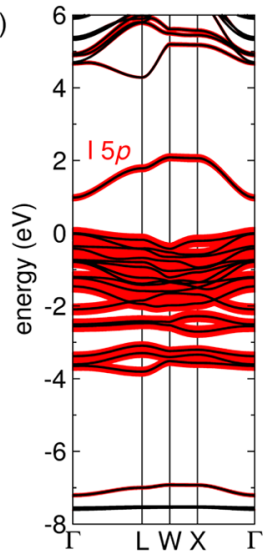

(b)

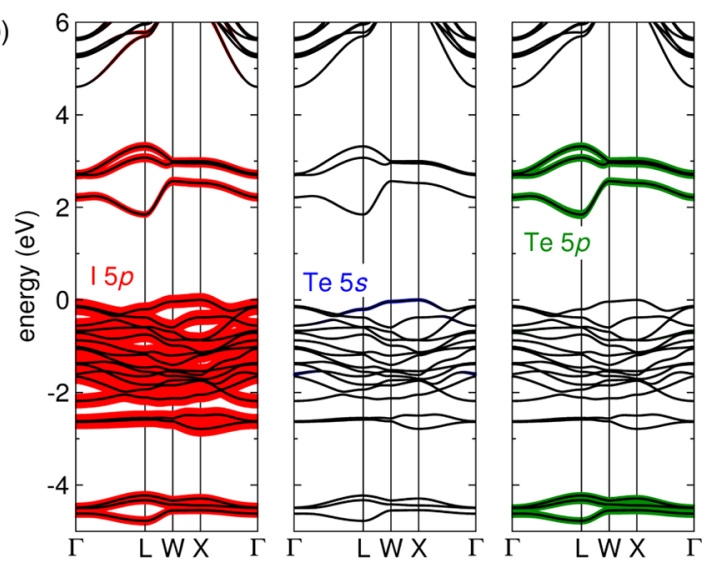

Figure 8. Band structures, including orbital projection analysis of (a) $\mathrm{Cs}_{2} \mathrm{SnI}_{6}$ and (b) $\mathrm{Cs}_{2} \mathrm{TeI}_{6}$, showing the contributions of the I $\mathrm{p}$ states in red, the $B 5$ s states in blue, and the $B 5 \mathrm{p}$ states in green.
$7 \mathrm{eV}$ below the highest occupied band due to the strong covalent interaction between $\mathrm{Sn}$ and $\mathrm{I}^{81}$ For $\mathrm{Cs}_{2} \mathrm{TeI}_{6}$, the conduction band is of antibonding Te $5 p$ and I $5 p$ character (Figure S9), consistent with the formal charge, Te(IV), that would have occupied $5 \mathrm{~s}$ and unoccupied $5 \mathrm{p}$ states. The occupied $5 \mathrm{~s}$ states in $\mathrm{Cs}_{2} \mathrm{TeI}_{6}$ are predominately localized around $E \approx-12 \mathrm{eV}$ but also have a small projection just below the Fermi energy.

From analysis of the calculated electronic structures, the band gaps and optical gaps in these materials are dictated by the energies of the empty $\mathrm{Sn} 5 \mathrm{~s}$ and Te $5 \mathrm{p}$ states at the lowest unoccupied band (as illustrated in Figure 9). The calculated

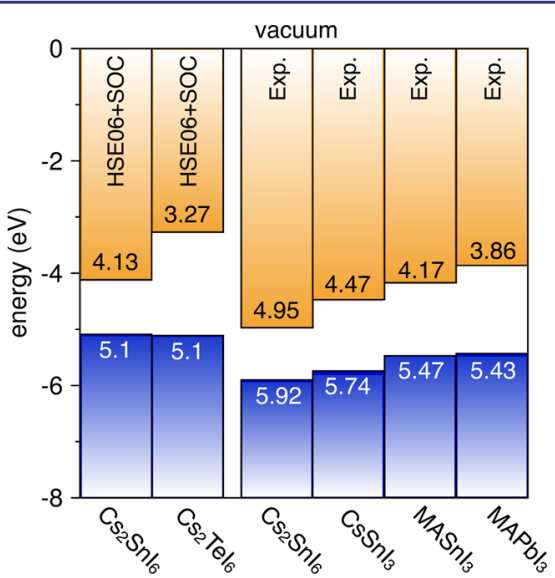

Figure 9. Comparison of DFT-calculated band alignments for $\mathrm{Cs}_{2} \mathrm{SnI}_{6}$ and $\mathrm{Cs}_{2} \mathrm{TeI}_{6}$ (HSE06+SOC) to experimentally determined band alignments. The experimental valence band maximum position of $\mathrm{Cs}_{2} \mathrm{SnI}_{6}$ was determined by XPS, and the conduction band minimum was positioned $0.97 \mathrm{eV}$ above (HSE06+SOC band gap). The experimental values are shown for $\mathrm{CsSnI}_{3},{ }^{63} \mathrm{MASnI}_{3},{ }^{83}$ and $\mathrm{MAPbI}_{3}{ }^{84}$ (where $\mathrm{MA}=\mathrm{CH}_{3} \mathrm{NH}_{3}^{+}$).

ionization potentials of both $\mathrm{Cs}_{2} \mathrm{SnI}_{6}$ and $\mathrm{Cs}_{2} \mathrm{TeI}_{6}$ are $\sim 5.1 \mathrm{eV}$, with the I $5 p$ states comprising the highest occupied bands, thus providing support that the valence band energy levels are pinned across the solid solution. The electron affinities (EA) of each material, 4.13 and $3.27 \mathrm{eV}$, respectively, are therefore controlled by the energy of the unoccupied states of Sn or Te. Density functional calculations of the band gaps across the solid solution $(25,50,75,88$, and $91 \% \mathrm{Te}$ concentration) are consistent with this notion and show a nearly linear increase in band gap across the solid solution (Figure S10). In contrast to the calculated band gaps, the experimentally determined optical gaps increase steadily from $x=0$ to $x=0.75$ until a rapid increase for $x=0.9$ and 1 . One possible explanation for the nonlinear trend in optical gaps arises from the nature of the optical transition compared to the fundamental band gap. As discussed previously, the DFT calculations reveal that the optical gap of $\mathrm{Cs}_{2} \mathrm{SnI}_{6}$ is derived from dipole-allowed transitions originating from below the valence band maximum. These transitions may persist across part of the solid solution, which would yield discrepancies between the experimental and calculated band gaps. Another possibility arises from the introduction of Te-derived states $\sim 0.85 \mathrm{eV}$ above the conduction band minimum upon substitution of Te for $\mathrm{Sn}$ in $\mathrm{Cs}_{2} \mathrm{SnI}_{6}$. These states may not immediately influence the magnitude of the band gap until the concentration of tellurium exceeds the percolation threshold for the face-centered cubic 
lattice $(12 \% \mathrm{Sn} ; 88 \% \mathrm{Te}){ }^{85}$ at which point the optical gap becomes reflective of the larger, indirect gap of $\mathrm{Cs}_{2} \mathrm{TeI}_{6}$.

$\mathrm{X}$-ray photoelectron spectroscopy (XPS) was performed to align the ionization potential with related perovskite halides. The ionization potential is -5.92 (5) eV (Figure S11), as shown in Figure 9, and is in good agreement with previous measurements of bulk powders of $\mathrm{Cs}_{2} \mathrm{SnI}_{6}\left(-5.40 \mathrm{eV}^{42}\right.$ and $\left.-5.94 \mathrm{eV}^{63}\right)$ but not with thin-film samples $\left(-6.1 \mathrm{eV}^{43}\right)$. Here, the conduction band was positioned using the $0.97 \mathrm{eV}$ band gap calculated using DFT (HSE06+SOC). The discrepancy between calculation and experiment is likely due to the extreme sensitivity of $\mathrm{Cs}_{2} \mathrm{SnI}_{6}$ to the nature of the exchange correlation functional used in the density functional calculations, as demonstrated in Figure S12, and/or from the surface sensitivity of the XPS measurements.

The band structures of both $\mathrm{Cs}_{2} \mathrm{SnI}_{6}$ and $\mathrm{Cs}_{2} \mathrm{TeI}_{6}$ present fairly dispersive conduction band states, indicative of significant orbital overlap despite the presence of ordered $B$-site vacancies. Calculation of the electron effective masses for $\mathrm{Cs}_{2} \mathrm{SnI}_{6}$ yield $m_{\mathrm{e}}^{*}=0.48 m_{0}$ (along $\Gamma \rightarrow \mathrm{X}$, in the $\left[\begin{array}{lll}0.5 & 0 & 0.5\end{array}\right]$ direction) and $m_{\mathrm{e}}^{*}=0.92 m_{0}\left(\Gamma \rightarrow \mathrm{L}\right.$, along $\left.\left[\begin{array}{lll}0.5 & 0.5 & 0.5\end{array}\right]\right)$, reflecting its intrinsic $n$-type behavior. The hole effective masses are considerably larger: $m_{\mathrm{h}}^{*}=1.32 m_{0}(\Gamma \rightarrow \mathrm{X})$ and $m_{\mathrm{h}}^{*}=2.75 m_{0}(\Gamma \rightarrow \mathrm{L})$. These calculations are consistent with recently reported calculations. ${ }^{43}$ $\mathrm{Cs}_{2} \mathrm{TeI}_{6}$ presents anisotropic electron and hole effective masses, with the lightest masses appearing along $\mathrm{L} \rightarrow \mathrm{W}\left(m_{\mathrm{m}}^{*}=0.22 m_{0}\right)$ and $\mathrm{X} \rightarrow \Gamma\left(m_{\mathrm{m}}^{*}=0.97 m_{0}\right)$ and the other directions producing significantly heavier charge carriers: $m_{\mathrm{m}}^{*}=1.40 m_{0}(\mathrm{~L} \rightarrow \Gamma)$ and $m_{\mathrm{m}}^{*}=4.40 m_{0}(\mathrm{X} \rightarrow \Gamma)$. The electron effective masses in these systems are all relatively light, albeit larger than those seen in the hybrid perovskites $\mathrm{CH}_{3} \mathrm{NH}_{3} \mathrm{PbI}_{3}$ and $\mathrm{CH}_{3} \mathrm{NH}_{3} \mathrm{SnI}_{3}\left(m_{\mathrm{m}}^{*}=\right.$ $0.15 m_{0}$ and $\left.0.28 m_{0}\right){ }^{18,86}$ Interestingly, unlike in the hybrid perovskites where the hole effective masses are smaller than the electron effective masses $\left(m_{\mathrm{m}}^{*}=0.12 m_{0}\right.$ and $0.13 m_{0}$ for $\mathrm{CH}_{3} \mathrm{NH}_{3} \mathrm{PbI}_{3}$ and $\mathrm{CH}_{3} \mathrm{NH}_{3} \mathrm{SnI}_{3}$, respectively); ${ }^{18,86}$ here, no such trend is observed. The lighter electron effective masses in $\mathrm{Cs}_{2} \mathrm{TeI}_{6}$ correlate with the reduced inter-octahedral distances observed in the crystal structures. The reduced electrical conductivity in tellurium-doped $\mathrm{Cs}_{2} \mathrm{SnI}_{6}$, despite the presence of dispersive conduction band states and small electron effective masses, suggests that the origin of this insulating behavior instead arises from the nature of carrier generation and mobility from intrinsic defects.

Intrinsic Defects. By varying the chemical potentials, $\mu_{i}$, as calculated from DFT, we can simulate the effect of experimentally varying the partial pressures in the formation of $\mathrm{Cs}_{2} \mathrm{SnI}_{6}$ and $\mathrm{Cs}_{2} \mathrm{TeI}_{6}$ and the resulting native defects. The accessible range of chemical potentials for $\mathrm{Cs}_{2} \mathrm{SnI}_{6}$ and $\mathrm{Cs}_{2} \mathrm{TeI}_{6}$, are shown in Figure $\mathrm{S} 13$ in a $2 \mathrm{D}\left(\mu_{\mathrm{Cs}}, \mu_{\mathrm{Te}}\right)$ plane. $^{87,88}$ Previous studies have performed similar calculations using the HSE functional but have adjusted the amount of nonlocal HF exchange $(\alpha)$ to fit the fundamental band gap to the experimental optical band gap, without consideration of the optical allowed transitions. However, as we have demonstrated, the fundamental band gap of $\mathrm{Cs}_{2} \mathrm{SnI}_{6}$ is dipole-disallowed, and HSE06 does not need to be altered to yield a reasonable description of the electronic structure of $\mathrm{Cs}_{2} \mathrm{SnI}_{6}$. As such, the close agreement between the calculated HSE06 electronic properties and the experimental optical band gap gives us confidence in our model.

Focusing on the formation of $n$-type defects to provide a theory-based understanding of the experimental data, calculations of $\mathrm{Cs}_{2} \mathrm{SnI}_{6}$ indicate that the formation energies of iodine vacancies $\left(V_{\mathrm{I}}\right)$ are small $(0.14-0.39 \mathrm{eV})$. Additionally, the $+1 / 0$ transition level is only $0.07 \mathrm{eV}$ below the lowest unoccupied band and is therefore likely to be the source of the native $n$-type conductivity observed in this material. While previous defect calculations of $\mathrm{Cs}_{2} \mathrm{SnI}_{6}$ have identified iodine vacancies as deep donor states with an ionization level of $0.52 \mathrm{eV},{ }^{44}$ the large size of the supercells used in our calculations as well as the improved accuracy of the fundamental band gap provide a representation of these defect states consistent with the intrinsic $n$-type conductivity and equilibrium carrier concentration on the order of $\sim 10^{16} \mathrm{~cm}^{-3}$. ${ }^{89} \mathrm{Cs}_{2} \mathrm{SnI}_{6}$ appears to be intrinsically doped and is tolerant of the native iodine vacancy defects.

$\mathrm{Cs}_{2} \mathrm{TeI}_{6}$, however, yields an electronic structure that is less amenable for introducing mobile carriers. The formation energy of $V_{\mathrm{I}}$ in $\mathrm{Cs}_{2} \mathrm{TeI}_{6}$ is much larger $(0.56-0.84 \mathrm{eV})$ than that in $\mathrm{Cs}_{2} \mathrm{SnI}_{6}$, which is likely responsible for the reduction in carrier concentration and insulating behavior upon tellurium substitution (Figure 10). The 2- to 4-fold increase in defect

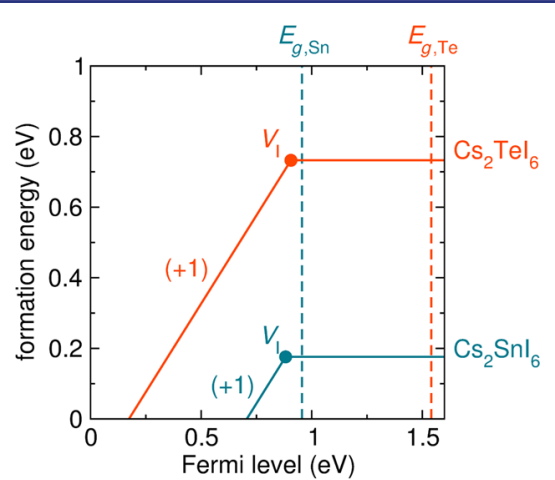

Figure 10. Formation energies for iodine vacancies in $\mathrm{Cs}_{2} \mathrm{SnI}_{6}$ (teal) and $\mathrm{Cs}_{2} \mathrm{TeI}_{6}$ (red), under tin/tellurium-poor conditions (point $\mathrm{C}$ in Figure S13). Sloped lines indicate the +1 charge state, and the solid dots represent the transitions levels $\epsilon\left(q / q^{\prime}\right)$. The dashed lines represent the fundamental band gap of each material.

formation enthalpy translates to a reduction in defect concentration by $\sim 10^{6}-10^{8}$ upon substitution of $\mathrm{Sn}$ by Te. Furthermore, the energy of the $(+1 / 0)$ transition level is effectively pinned across the solid solution at $E=0.9 \mathrm{eV}$ above $E_{\mathrm{F}}$. Therefore, the depth of the defect level is largely dependent upon the energy of the empty $B$ states that make up the lowest unoccupied bands. In $\mathrm{Cs}_{2} \mathrm{SnI}_{6}, V_{\mathrm{I}}$ defect states form $\sim 0.07 \mathrm{eV}$ below the unoccupied $\mathrm{Sn} 5 \mathrm{~s}$ states and therefore can behave as donor states. In $\mathrm{Cs}_{2} \mathrm{TeI}_{6}$, the defect transition level is deep in the band gap, with the lowest unoccupied band $\sim 0.85 \mathrm{eV}$ higher in energy, such that the native defects are likely to behave as deep trap states for charge carriers. These dramatic changes can account for the reduction in carrier concentration, mobility, and conductivity upon tellurium substitution.

The general structure-property relationships in these materials follow from the close-packed anionic lattice and the interaction between the $B$-site ions and the halides. The main difference between the electronic band structures of $\mathrm{Cs}_{2} \mathrm{SnI}_{6}$ and $\mathrm{Cs}_{2} \mathrm{TeI}_{6}$ is the position and symmetry of the conduction band because the valence bands are pinned by the I $5 p$ states. The increased Pauling electronegativity of $\mathrm{Te}^{4+}(\chi=2.1)$ compared to that of $\mathrm{Sn}^{4+}(\chi=1.8)^{90}$ increases the covalency of the $\left[\mathrm{TeI}_{6}\right]$ octahedral units relative to that of $\left[\mathrm{SnI}_{6}\right]$. This notion is further supported by Bader charge analysis of $\mathrm{Cs}_{2} \mathrm{SnI}_{6}$ 
Table 3. Bader Charges Generated for $\mathrm{Cs}_{2} \mathrm{SnI}_{6}$ and $\mathrm{Cs}_{2} \mathrm{TeI}_{6}$

\begin{tabular}{cccc} 
& $\mathrm{Cs}$ & $B$ & $\mathrm{I}$ \\
$\mathrm{Cs}_{2} \mathrm{SnI}_{6}$ & 0.84 & 1.31 & -0.50 \\
$\mathrm{Cs}_{2} \mathrm{TeI}_{6}$ & 0.84 & 0.89 & -0.43 \\
\hline
\end{tabular}

and $\mathrm{Cs}_{2} \mathrm{TeI}_{6}$ (Table 3), which shows reduced Bader charges for Te relative to $\mathrm{Sn}$. The average integrated COHP (ICOHP) value for each $B-I$ bond was found to be -3.4 and -2.0 for $\mathrm{Cs}_{2} \mathrm{SnI}_{6}$ and $\mathrm{Cs}_{2} \mathrm{TeI}_{6}$. This would suggest a more covalent interaction for the $\mathrm{Sn}-\mathrm{I}$ bonds relative to the Te-I bonds; however, we note that this computational result likely results from the shorter bond lengths in $\mathrm{Cs}_{2} \mathrm{SnI}_{6}$. The Born effective charges for the $B$-site cation in $\mathrm{Cs}_{2} \mathrm{SnI}_{6}$ and $\mathrm{Cs}_{2} \mathrm{TeI}_{6}$ (shown in Table SII) are 3.9 and 4.4, respectively, which indicate a higher degree of covalency for the $\mathrm{Te}-\mathrm{I}$ bonds ${ }^{91}$ in support of the Bader charge analysis and the Pauling electronegativities.

While the close-packed iodine lattice is responsible for the dispersive conduction band states, increased covalency of the $\mathrm{Te}-\mathrm{I}$ bonds relative to $\mathrm{Sn}-\mathrm{I}$ affect the electronic structure and defect chemistry in these materials. The increased covalency of $\mathrm{Te}-\mathrm{I}$ bonding likely prevents the formation of iodine vacancies in the material and raises the energy of the conduction band, which yields defect states that form deep within the band gap. While the smaller band gap and more ionic $\left[\mathrm{SnI}_{6}\right]$ units in $\mathrm{Cs}_{2} \mathrm{SnI}_{6}$ yield a defect-tolerant material with shallow donor states, the larger band gap and more molecular $\left[\mathrm{TeI}_{6}\right]$ units render the material intolerant to crystallographic defects.

\section{CONCLUSIONS}

Vacancy-ordered double perovskite halides present a fertile testbed to explore structure-property relationships in complex semiconductors with potential for transformative applications in photovoltaics. From the preparation of the solid-solution of $\mathrm{Cs}_{2} \mathrm{Sn}_{1-x} \mathrm{Te}_{x} \mathrm{I}_{6}$, the end member, $\mathrm{Cs}_{2} \mathrm{SnI}_{6}$, exhibits intrinsic $n$ type conductivity, yet substitution of tin by tellurium is accompanied by a reduction in conductivity, carrier concentration, and carrier mobility. The poor conductivity of $\mathrm{Cs}_{2} \mathrm{TeI}_{6}$ is attributed mostly to the electronic structure and hindered formation of intrinsic iodine vacancy donor defects, despite a predicted increase in carrier mobility, as further supported from hybrid-functional DFT calculations. This conclusion is based upon extensive structural characterization by high-resolution time-of-flight neutron powder diffraction, synchrotron powder $\mathrm{X}$-ray diffraction, and X-ray pair distribution function analysis, which together reveal that tellurium does not induce structural distortions or instabilities across this series of materials as one might hypothesize from the potential for stereochemical activity of the formal $5 s^{2}$ valence electron configuration. Despite the absence of $3 \mathrm{D}$ covalent $B-X-B$ connectivity in vacancyordered double perovskite crystal structure, the close-packed anionic lattice forms dispersive frontier electronic bands with light carriers, provided that the bonding environment between $B$ - and $X$-site ions can stabilize the highly symmetric structure and stabilize shallow defect states to provide mobile charge carriers, as is important for the large and transformative family of perovskite halide semiconductors.

\section{MATERIALS AND METHODS}

Synthesis of $\mathrm{Snl}_{4}$ and $\mathrm{Tel}_{4}$. Two equivalents of solid $\mathrm{I}_{2}$ were added to one equivalent of either elemental $\mathrm{Sn}$ or Te in a silica ampule and sealed under dynamic vacuum. The reaction was heated to $200{ }^{\circ} \mathrm{C}$ until the purple iodine vapor in the tube had subsided $(\sim 60 \mathrm{~h})$, resulting in bright orange $\left(\mathrm{SnI}_{4}\right)$ and dark gray $\left(\mathrm{TeI}_{4}\right)$ products, as confirmed with powder X-ray diffraction. Yields are quantitative.

Synthesis of $\mathrm{Cs}_{2} \mathrm{Sn}_{1-x} \mathrm{Te}_{x} \mathrm{I}_{6}$. The compounds in the solid-solution series $\mathrm{Cs}_{2} \mathrm{Sn}_{1-x} \mathrm{Te}_{x} \mathrm{I}_{6}$ were synthesized by modification of the method described by Lee et al. ${ }^{42}$ In a beaker, $\sim 0.5 \mathrm{mmol}$ of $\mathrm{Cs}_{2} \mathrm{CO}_{3}$ was added to $3 \mathrm{~mL}$ of $57 \%$ hydriodic acid (aqueous, $1.5 \% \mathrm{H}_{3} \mathrm{PO}_{2}$ ) and stirred to dissolve. Solid $\mathrm{Cs}_{2} \mathrm{CO}_{3}$ was added slowly to hydroiodic acid in order to account for the vigorous reaction between $\mathrm{Cs}_{2} \mathrm{CO}_{3}$ and $\mathrm{HI}$, which produces heat and gaseous $\mathrm{CO}_{2}$. In a separate beaker, the appropriate ratio of $\mathrm{SnI}_{4} / \mathrm{TeI}_{4}$ precursor was dissolved in $15 \mathrm{~mL}$ of absolute ethanol and $2 \mathrm{~mL}$ of $57 \%$ hydriodic acid (aqueous, $1.5 \% \mathrm{H}_{3} \mathrm{PO}_{2}$ ), to which excess elemental iodine was added to consume the hypophosphorus acid stabilizing agent. The beaker containing the $\mathrm{BI}_{4}$ precursors was gently heated to $T \approx 60^{\circ} \mathrm{C}$ to encourage solubility. Once the solids had completely dissolved into their respective solutions, the $\mathrm{Cs}_{2} \mathrm{CO}_{3} / \mathrm{HI}$ solution was quickly added to the $\mathrm{BI}_{4}$ solution, resulting in a black precipitate that immediately crashed out of solution. The reaction was stirred for an additional $30 \mathrm{~min}$. The precipitate was collected by centrifugation and washed three times with absolute ethanol. The final products were dried at $60^{\circ} \mathrm{C}$ for $24 \mathrm{~h}$. Phase purity was verified by powder X-ray diffraction. Synthetic yields range from $87-94 \%$ for the solid solution, as determined by comparing the amount of product collected from the synthesis to the theoretical mass of product calculated from precursor amounts.

Characterization. Powder X-ray diffraction data were collected on a Scintag X-2 Diffractometer with $\mathrm{CuK} \alpha$ radiation. Diffraction data were analyzed using the Rietveld method as implemented in GSAS/ EXPGUI. ${ }^{92,93}$ High-resolution time-of-flight neutron powder diffraction was performed on the POWGEN diffractometer at the Spallation Neutron Source, Oak Ridge National Laboratory at $T=300 \mathrm{~K}$ and $T=$ $10 \mathrm{~K}$. High-resolution synchrotron powder X-ray diffraction data were collected on the 11-BM-B beamline at the Advanced Photon Source, Argonne National Laboratory. ${ }^{94} \mathrm{X}$-ray pair distribution function data were calculated from X-ray total scattering collected at the 11-ID-B beamline at the Advanced Photon Source, Argonne National Laboratory. 2D diffraction images were calibrated and radially integrated using the FIT2D software. ${ }^{95}$ The PDFs were extracted using PDFgetX3 after correcting for the empty Kapton capillary container $^{96}$ and were quantitatively modeled using PDFgui. ${ }^{97}$ The crystal structures were visualized using VESTA. ${ }^{98}$

Electrical resistivity measurements were performed using a Physical Properties Measurement System (Quantum Design, Inc.) on coldpressed pellets of the members of the solid-solution series using a 4wire configuration with Au-paste contacts and Pt wires. Measurements were performed upon cooling and then again upon heating to ensure reproducibility. Analysis of the temperature dependent resistivity data was performed as in ref 99 . Hall measurements were performed using a Physical Properties Measurement System (Quantum Design, Inc.) in the van der Pauw configuration on cold-pressed polycrystalline pellets. Measurements on the Te-substituted samples were not possible due to the highly insulating nature of the specimens.

UV-visible diffuse reflectance spectroscopy was performed on powdered samples of the solid-solution series diluted to $10 \mathrm{wt} \%$ in $\mathrm{BaSO}_{4}$, using $\mathrm{BaSO}_{4}$ as a baseline; spectra were acquired using a Thermo Nicolet Evolution 300 spectrophotometer with a Praying Mantis mirror setup from $\lambda=200$ to $1000 \mathrm{~nm}$ at a scan rate of 240 $\mathrm{nm} / \mathrm{min}$. Heat capacity measurements were performed on pelleted samples of $\mathrm{Cs}_{2} \mathrm{SnI}_{6}$ and $\mathrm{Cs}_{2} \mathrm{TeI}_{6}$ using the quasi-adiabatic heat-pulse technique implemented in the Quantum Design, Inc. PPMS at $T=3-$ $150 \mathrm{~K}$.

Photoluminescence (PL) spectra were collected from cold-pressed polycrystalline pellets of $\mathrm{Cs}_{2} \mathrm{SnI}_{6}$ and $\mathrm{Cs}_{2} \mathrm{TeI}_{6}$ mounted on glass substrates. The compounds were excited with an LED head at $780 \mathrm{~nm}$. The excitation beam was modulated at $50 \mathrm{~Hz}$ and loosely focused (spot size roughly $0.8 \mathrm{~cm}$ in diameter) on the samples. The incident angle of the excitation beam was $\sim 45^{\circ}$, and the resulting PL were collected at an angle perpendicular to the excitation beam and detected by an amplified Ge photodiode routed to a lock-in amplifier. The $830 \mathrm{~nm}$ long-pass filters were placed before the detector to 
remove excitation light. The PL spectra were corrected for monochromator and detector efficiencies using a calibrated lamp.

The XPS $(h \nu=1486.7 \mathrm{eV})$ measurements for $\mathrm{Cs}_{2} \mathrm{SnI}_{6}$ were performed on a PHI 5600 XPS instrument, which has been previously described in detail. ${ }^{100}$ For the XPS core level, valence band, and workfunction measurements, the pass energy was set to $11.75,5.85$, and $2.95 \mathrm{eV}$ with a step size of $0.10,0.050$, and $0.025 \mathrm{eV}$, respectively. The spectrometer was calibrated with metallic Fermi edges and atomic core levels from $\mathrm{Au}, \mathrm{Ag}, \mathrm{Cu}$, or $\mathrm{Mo}$. The instrumental response of the XPS spectrometer was $350 \mathrm{meV}$ and is determined from comparing the measured and calculated Fermi function for a particular instrument condition. The energy uncertainty of XPS $( \pm 0.050 \mathrm{eV})$ is a statistical quantity determined from multiple measurements of the Fermi energy. The $\mathrm{Cs}_{2} \mathrm{SnI}_{6}$ pellet did not charge as verified with power-dependent measurements. To determine the $E_{\mathrm{F}}-E_{\mathrm{VBM}}$ value, we used two methods. First, we fit both the baseline and main valence band feature to a line, and the intersection was taken as the onset value, $E_{\mathrm{F}}-E_{\mathrm{VBM}}$ $=1.44 \pm 0.05 \mathrm{eV}$. Second, we used the calculated density of states (DOS). We broaden the DOS with a $350 \mathrm{meV}$ fwhm Gaussian line shape, which is the instrument response of the spectrometer. Because the DOS calculation does not account for the XPS cross-section, we only fit the broadened DOS to the rising edge of the experimental spectrum, similar to that used by Kraut et al. and Chambers et al., $E_{\mathrm{F}}-$ $E_{\mathrm{VBM}}=1.49 \pm 0.05 \mathrm{eV} .^{101,102}$

Density Functional Theory Calculations. First-principles calculations were performed using the Vienna $A b$ initio Simulation Package (VASP), ${ }^{7-76}$ a periodic density functional theory (DFT) code utilizing a plane-wave basis set, with interactions between the core and valence electrons described using the projector augmented wave (PAW) method. ${ }^{103}$ This study employed the HSE06 functional, ${ }^{77}$ which combines $75 \%$ exchange and $100 \%$ of the correlation energies from the Perdew, Burke, and Ernzerhof (PBE) functional ${ }^{104}$ together with $25 \%$ exact HF exchange at short ranges. HSE06 has been shown to accurately reproduce the lattice constants and electronic properties of a wide range of solid-state semiconductors. ${ }^{105-107}$ For band structure and density of states calculations, special attention was paid to accurately modeling the relativistic effects seen in $\mathrm{Sn}, \mathrm{Te}$, and I, through use of scalar relativistic PAW pseudopotentials and explicit treatment of spin-orbit coupling (SOC) effects. ${ }^{108} \mathrm{~A}$ plane wave cutoff of $350 \mathrm{eV}$ and $k$-point sampling of $\Gamma$ centered $3 \times 3 \times 3$ for the 9-atom unit cells of $\mathrm{Cs}_{2} \mathrm{SnI}_{6}$ and $\mathrm{Cs}_{2} \mathrm{TeI}_{6}$ were used. For structural relaxations the ionic forces were converged to $0.01 \mathrm{eV} \AA^{-1}$, we used a larger cutoff energy $(455 \mathrm{eV})$ in order to avoid errors arising from Pulay stress. The HSE06 optimized crystal structures can be found online in a public repository. ${ }^{109}$

Electronic structure calculations of the solid solution were calculated by constructing a $2 \times 2 \times 2$ supercell (containing 288 atoms) of the $\mathrm{Cs}_{2} \mathrm{SnI}_{6}$ standard $F m \overline{3} m$ unit cell. Compositions corresponding to $25,50,75,88$, and $91 \% \mathrm{Te}$ concentration were generated based on the quasi-random structure (SQS) approach, ${ }^{107,110}$ in which an appropriate number of $\mathrm{Sn}$ atoms are replaced with Te $(8$, $16,24,28$, and 29 atoms, respectively). Due to the solid-solution nature of the alloys observed by experiment, these random structures are expected to demonstrate properties representative of each composition. Each structure was geometrically relaxed using the HSE06 functional at a single $k$-point before the band gap was calculated using HSE06+SOC.

Phonon calculations were carried out on $2 \times 2 \times 2$ supercells of the standard cell structures of $\mathrm{Cs}_{2} \mathrm{SnI}_{6}$ and $\mathrm{Cs}_{2} \mathrm{TeI}_{6}$, containing 288 atoms. These calculations were performed at a single $k$-point $(\Gamma)$ and employed the PBEsol functional without the use of SOC; explicit treatment of relativistic effects greatly increases computational complexity and has been shown to have a minimal effect on lattice vibrations. ${ }^{111,112}$ Lattice-dynamics calculations were carried out using the Phonopy package, ${ }^{113}$ using the finite difference approach to calculate the interatomic force constants (IFCs). Dielectric constants and Born effective charges were calculated within the framework of density functional perturbation theory (DFPT) at a denser $k$-point mesh of $4 \times 4 \times 4$, using the PBEsol functional both with and without SOC effects. These were used to make nonanalytical corrections to the calculated phonon frequencies, in order to determine the splitting between the longitudinal and transverse optical phonons (LO-TO splitting).

COHPs were calculated using the LOBSTER program based on wavefunctions calculated using HSE06. ${ }^{82,114}$ The optical transition matrix elements were calculated using the transversal approximation and used to construct the imaginary dielectric function and corresponding optical absorption. ${ }^{115}$ Band alignments were performed using a slab model (45 ̊̊ slab, $35 \AA$ \&acuum), with the corresponding electrostatic potential averaged along the $c$-direction, using the MacroDensity package. ${ }^{116-118}$ The energy of the potential at the plateau was used as the external vacuum level. The slab calculations were performed using the HSE06, PBE, and PBEsol functionals, with corrections to the valence band energy and band gap in each case taken from the HSE06+SOC, PBE+SOC, and PBEsol+SOC calculated bulks. All defects were calculated using HSE06 in a $3 \times 3 \times 3(243$ atom) supercell, employing a single $k$-point at $\Gamma$. All the competing phases required to construct the chemical potential limits were structurally converged using HSE06. Bader charge analysis was calculated using the bader code ${ }^{119,120}$ with the GPU port of VASP $^{121,122}$ using the HSE06 functional and explicit treatment of spin-orbit coupling.

\section{ASSOCIATED CONTENT}

Supporting Information

The Supporting Information is available free of charge on the ACS Publications website at DOI: 10.1021/jacs.6b03207.

Additional neutron powder diffraction data, low-temperature specific heat data, X-ray pair distribution function analysis data, X-ray photoelectron spectroscopy data, additional UV-visible diffuse reflectance and photoluminescence spectra, and computational details including DFT-calculated band gaps, band alignments, LO-TO splitting values, dielectric constants and Born effective charges, and chemical potential diagrams (PDF) Crystallographic information file for $\mathrm{Cs}_{2} \mathrm{Sn}_{0.25} \mathrm{Te}_{0.75} \mathrm{I}_{6}$ (CIF)

Crystallographic information file for $\mathrm{Cs}_{2} \mathrm{Sn}_{0.9} \mathrm{Te}_{0.1} \mathrm{I}_{6}$ (CIF)

Crystallographic information file for $\mathrm{Cs}_{2} \mathrm{Sn}_{0.5} \mathrm{Te}_{0.5} \mathrm{I}_{6}$ (CIF)

Crystallographic information file for $\mathrm{Cs}_{2} \mathrm{Sn}_{0.1} \mathrm{Te}_{0.9} \mathrm{I}_{6}$ (CIF)

Crystallographic information file for $\mathrm{Cs}_{2} \mathrm{SnI}_{6}$ (CIF)

Crystallographic information file for $\mathrm{Cs}_{2} \mathrm{TeI}_{6}$ (CIF)

Crystallographic information file for $\mathrm{Cs}_{2} \mathrm{Sn}_{0.75} \mathrm{Te}_{0.25} \mathrm{I}_{6}$ (CIF)

\section{AUTHOR INFORMATION}

\section{Corresponding Author}

*E-mail: james.neilson@colostate.edu.

\section{Notes}

The authors declare no competing financial interest.

\section{ACKNOWLEDGMENTS}

We thank M. M. Reynolds for use of her optical spectrometer, Y. Yang for photoluminescence measurements, A. Huq for neutron powder diffraction measurements, and J. A. Kurzman, A. J. Martinolich, and B. C. Melot for useful discussions. Research at the 11-ID-B and 11-BM-B beamlines used resources of the Advanced Photon Source, a U.S. Department of Energy (DOE) Office of Science User Facility operated for the DOE Office of Science by Argonne National Laboratory under Contract No. DE-AC02-06CH11357. A portion of this 
research at ORNL's Spallation Neutron Source was sponsored by the Scientific User Facilities Division, Office of Basic Energy Sciences, U.S. Department of Energy. The calculations performed at CSU used the CSU ISTeC Cray HPC System supported by NSF Grant No. CNS-0923386 and the Extreme Science and Engineering Discovery Environment (XSEDE), which is supported by National Science Foundation grant number ACI-1053575. This work also made use of the ARCHER UK National Supercomputing Service (http:// www.archer.ac.uk), via the membership of the UK's HPC Materials Chemistry Consortium, which is funded by EPSRC (EP/L000202). The work at UCL was supported by EPSRC (EP/N01572X/1). D.O.S. acknowledges support from the SUPERSOLAR Solar Energy Hub (EP/J017361/1) for the provision of a flexible funding call award. A.M.G. acknowledges Diamond Light Source for the cosponsorship of a studentship on the EPSRC Centre for Doctoral Training in Molecular Modelling and Materials Science (EP/L015862/1). We gratefully acknowledge the support of NVIDIA Corporation with the donation of the Tesla K40 GPU used for this research. E.M.M. would like to thank U.S. Department of Energy, Office of Science, Office of Basic Energy Sciences, Division of Chemical Sciences, Geosciences, and Biosciences (contract DEAC36- 08GO28308).

\section{REFERENCES}

(1) Kagan, C.; Mitzi, D.; Dimitrakopoulos, C. Science 1999, 286, 945-947.

(2) Labram, J. G.; Fabini, D. H.; Perry, E. E.; Lehner, A. J.; Wang, H.; Glaudell, A. M.; Wu, G.; Evans, H.; Buck, D.; Cotta, R.; Echegoyen, L.; Wudl, F.; Seshadri, R.; Chabinyc, M. L. J. Phys. Chem. Lett. 2015, 6, 3565-3571.

(3) Dohner, E. R.; Jaffe, A.; Bradshaw, L. R.; Karunadasa, H. I. J. Am. Chem. Soc. 2014, 136, 13154-13157.

(4) Protesescu, L.; Yakunin, S.; Bodnarchuk, M. I.; Krieg, F.; Caputo, R.; Hendon, C. H.; Yang, R. X.; Walsh, A.; Kovalenko, M. V. Nano Lett. 2015, 15, 3692-3696.

(5) Kojima, A.; Teshima, K.; Shirai, Y.; Miyasaka, T. J. Am. Chem. Soc. 2009, 131, 6050-6051.

(6) Chung, I.; Song, J.-H.; Im, J.; Androulakis, J.; Malliakas, C. D.; Li, H.; Freeman, A. J.; Kenney, J. T.; Kanatzidis, M. G. J. Am. Chem. Soc. 2012, 134, 8579-8587.

(7) Gao, P.; Grätzel, M.; Nazeeruddin, M. K. Energy Environ. Sci. 2014, 7, 2448-2463.

(8) Colella, S.; Mosconi, E.; Fedeli, P.; Listorti, A.; Gazza, F.; Orlandi, F.; Ferro, P.; Besagni, T.; Rizzo, A.; Calestani, G.; Gigli, G.; De Angelis, F.; Mosca, R. Chem. Mater. 2013, 25, 4613-4618.

(9) Eperon, G. E.; Paternò, G. M.; Sutton, R. J.; Zampetti, A.; Haghighirad, A. A.; Cacialli, F.; Snaith, H. J. J. Mater. Chem. A 2015, 3, 19688-19695

(10) Chung, I.; Lee, B.; He, J.; Chang, R. P.; Kanatzidis, M. G. Nature 2012, 485, 486-489.

(11) Lee, M. M.; Teuscher, J.; Miyasaka, T.; Murakami, T. N.; Snaith, H. J. Science 2012, 338, 643-647.

(12) Liu, M.; Johnston, M. B.; Snaith, H. J. Nature 2013, 501, 395398.

(13) Burschka, J.; Pellet, N.; Moon, S.-J.; Humphry-Baker, R.; Gao, P.; Nazeeruddin, M. K.; Grätzel, M. Nature 2013, 499, 316-319.

(14) Choi, J. J.; Yang, X.; Norman, Z. M.; Billinge, S. J.; Owen, J. S. Nano Lett. 2014, 14, 127-133.

(15) Fabini, D. J. Phys. Chem. Lett. 2015, 6, 3546-3548.

(16) Babayigit, A.; Ethirajan, A.; Muller, M.; Conings, B. Nat. Mater. 2016, 15, 247-251.

(17) Stoumpos, C. C.; Malliakas, C. D.; Kanatzidis, M. G. Inorg. Chem. 2013, 52, 9019-9038.
(18) Frost, J. M.; Butler, K. T.; Brivio, F.; Hendon, C. H.; van Schilfgaarde, M.; Walsh, A. Nano Lett. 2014, 14, 2584-2590.

(19) Bass, K. K.; McAnally, R. E.; Zhou, S.; Djurovich, P. I.; Thompson, M. E.; Melot, B. C. Chem. Commun. 2014, 50, 1581915822.

(20) Wozny, S.; Yang, M.; Nardes, A. M.; Mercado, C. C.; Ferrere, S.; Reese, M. O.; Zhou, W.; Zhu, K. Chem. Mater. 2015, 27, 4814-4820.

(21) Mosconi, E.; Azpiroz, J. M.; De Angelis, F. Chem. Mater. 2015, 27, 4885-4892.

(22) Eames, C.; Frost, J. M.; Barnes, P. R.; O'regan, B. C.; Walsh, A.; Islam, M. S. Nat. Commun. 2015, 6, 7497.

(23) Leijtens, T.; Eperon, G. E.; Pathak, S.; Abate, A.; Lee, M. M.; Snaith, H. J. Nat. Commun. 2013, 4, 2885.

(24) Deretzis, I.; Alberti, A.; Pellegrino, G.; Smecca, E.; Giannazzo, F.; Sakai, N.; Miyasaka, T.; La Magna, A. Appl. Phys. Lett. 2015, 106, 131904.

(25) Bertoluzzi, L. J. Phys.: Conf. Ser. 2015, 609, 012001.

(26) Sadoughi, G.; Starr, D. E.; Handick, E.; Stranks, S. D.; Gorgoi, M.; Wilks, R. G.; Baer, M.; Snaith, H. J. ACS Appl. Mater. Interfaces 2015, 7, 13440-13444.

(27) Attfield, J. P.; Lightfoot, P.; Morris, R. E. Dalton Trans. 2015, 44, 10541-10542.

(28) Amat, A.; Mosconi, E.; Ronca, E.; Quarti, C.; Umari, P.; Nazeeruddin, M. K.; Grätzel, M.; De Angelis, F. Nano Lett. 2014, 14, $3608-3616$.

(29) Møller, C. K. Nature 1958, 182, 1436-1436.

(30) Knop, O.; Wasylishen, R. E.; White, M. A.; Cameron, T. S.; Oort, M. J. M. V. Can. J. Chem. 1990, 68, 412-422.

(31) Swainson, I. P.; Stock, C.; Parker, S. F.; Van Eijck, L.; Russina, M.; Taylor, J. W. Phys. Rev. B: Condens. Matter Mater. Phys. 2015, 92, 100303.

(32) Anderson, M. T.; Greenwood, K. B.; Taylor, G. A.; Poeppelmeier, K. R. Prog. Solid State Chem. 1993, 22, 197-233.

(33) McClure, E. T.; Ball, M. R.; Windl, W.; Woodward, P. M. Chem. Mater. 2016, 28, 1348-1354.

(34) Slavney, A. H.; Hu, T.; Lindenberg, A. M.; Karunadasa, H. I. J. Am. Chem. Soc. 2016, 138, 2138-2141.

(35) Rössler, K.; Winter, J. Chem. Phys. Lett. 1977, 46, 566-570.

(36) Henkel, W.; Pelzl, J.; Höck, K.; Thomas, H. Z. Phys. B: Condens. Matter Quanta 1980, 37, 321-332.

(37) Brendel, W.; Samartzis, T.; Brendel, C.; Krebs, B. Thermochim. Acta 1985, 83, 167-172.

(38) Abrahams, S.; Ihringer, J.; Marsh, P. Acta Crystallogr., Sect. B: Struct. Sci. 1989, 45, 26-34.

(39) Kieslich, G.; Sun, S.; Cheetham, A. K. Chem. Sci. 2014, 5, 47124715.

(40) Kieslich, G.; Sun, S.; Cheetham, T. Chem. Sci. 2015, 6, 34303433.

(41) Brown, I. D. Can. J. Chem. 1964, 42, 2758-2767.

(42) Lee, B.; Stoumpos, C. C.; Zhou, N.; Hao, F.; Malliakas, C.; Yeh, C.-Y.; Marks, T. J.; Kanatzidis, M. G.; Chang, R. P. J. Am. Chem. Soc. 2014, 136, 15379-15385.

(43) Saparov, B.; Sun, J.-P.; Meng, W.; Xiao, Z.; Duan, H.-S.; Gunawan, O.; Shin, D.; Hill, I. G.; Yan, Y.; Mitzi, D. B. Chem. Mater. 2016, 28, 2315-2322.

(44) Xiao, Z.; Zhou, Y.; Hosono, H.; Kamiya, T. Phys. Chem. Chem. Phys. 2015, 17, 18900-18903.

(45) Zakutayev, A.; Caskey, C. M.; Fioretti, A. N.; Ginley, D. S.; Vidal, J.; Stevanovic, V.; Tea, E.; Lany, S. J. Phys. Chem. Lett. 2014, 5, $1117-1125$

(46) Brandt, R. E.; Stevanović, V.; Ginley, D. S.; Buonassisi, T. MRS Commun. 2015, 5, 265-275.

(47) Walsh, A.; Scanlon, D. O.; Chen, S.; Gong, X.; Wei, S.-H. Angew. Chem. 2015, 127, 1811-1814.

(48) Jansen, M.; Wedig, U. Angew. Chem., Int. Ed. 2008, 47, 1002610029 .

(49) Aullón, G.; Alvarez, S. Theor. Chem. Acc. 2009, 123, 67-73.

(50) Limpinsel, M.; Farhi, N.; Berry, N.; Lindemuth, J.; Perkins, C. L.; Lin, Q.; Law, M. Energy Environ. Sci. 2014, 7, 1974-1989. 
(51) Shklovskii, B. L.; Efros, A. L. In Electronic Properties of Doped Semiconductors; Cardona, M., Fulde, P., Queisser, H. J., Eds.; Springer Series in Solid-State Sciences No. 45; Springer: New York, 1984.

(52) King, G.; Abakumov, A. M.; Woodward, P. M.; Llobet, A.; Tsirlin, A. A.; Batuk, D.; Antipov, E. V. Inorg. Chem. 2011, 50, 77927801.

(53) Maughan, A. E.; Kurzman, J. A.; Neilson, J. R. Inorg. Chem. 2015, 54, 370-378.

(54) Shannon, R. t.; Prewitt, C. T. Acta Crystallogr., Sect. B: Struct. Crystallogr. Cryst. Chem. 1969, 25, 925-946.

(55) Shannon, R.; Prewitt, C. Acta Crystallogr., Sect. B: Struct. Crystallogr. Cryst. Chem. 1970, 26, 1046-1048.

(56) Shannon, R. D. Acta Crystallogr., Sect. A: Cryst. Phys., Diffr., Theor. Gen. Crystallogr. 1976, 32, 751-767.

(57) Swanson, B. Phys. Status Solidi A 1978, 47, K95-K98.

(58) Kaltzoglou, A.; Antoniadou, M.; Kontos, A. G.; Stoumpos, C. C.; Perganti, D.; Siranidi, E.; Raptis, V.; Trohidou, K. N.; Psycharis, V.; Kanatzidis, M. G.; Falaras, P. J. Phys. Chem. C 2016, 120, 11777.

(59) Bencherif, Y.; Boukra, A.; Zaoui, A.; Ferhat, M. Infrared Phys. Technol. 2011, 54, 39-43.

(60) Waghmare, U.; Spaldin, N.; Kandpal, H.; Seshadri, R. Phys. Rev. B: Condens. Matter Mater. Phys. 2003, 67, 125111.

(61) Zhong, W.; King-Smith, R.; Vanderbilt, D. Phys. Rev. Lett. 1994, 72, 3618.

(62) Dolgonos, A.; Mason, T. O.; Poeppelmeier, K. R. J. Solid State Chem. 2016, 240, 43-48.

(63) Zhang, J.; Yu, C.; Wang, L.; Li, Y.; Ren, Y.; Shum, K. Sci. Rep. 2014, 4, 6954-6959.

(64) Peresh, E. Y.; Zubaka, O.; Sidei, V.; Barchii, I.; Kun, S.; Kun, A. Inorg. Mater. 2002, 38, 859-863.

(65) Ranfagni, A.; Mugnai, D.; Bacci, M.; Viliani, G.; Fontana, M. Adv. Phys. 1983, 32, 823-905.

(66) Blasse, G.; Dirksen, G.; Abriel, W. Chem. Phys. Lett. 1987, 136, 460-464.

(67) Drummen, P.; Donker, H.; Smit, W.; Blasse, G. Chem. Phys. Lett. 1988, 144, 460-462.

(68) Donker, H.; Smit, W.; Blasse, G. J. Phys. Chem. Solids 1989, 50, 603-609.

(69) Zunger, A.; Jaffe, J. Phys. Rev. Lett. 1983, 51, 662-665.

(70) Bellaiche, L.; Wei, S.-H.; Zunger, A. Phys. Rev. B: Condens. Matter Mater. Phys. 1996, 54, 17568.

(71) Wu, J.; Walukiewicz, W.; Yu, K.; Ager, J., III; Haller, E.; Miotkowski, I.; Ramdas, A.; Su, C.-H.; Sou, I.; Perera, R.; Denlinger, J. Phys. Rev. B: Condens. Matter Mater. Phys. 2003, 67, 035207.

(72) Wu, J.; Walukiewicz, W.; Yu, K.; Denlinger, J.; Shan, W.; Ager, J., III; Kimura, A.; Tang, H.; Kuech, T. Phys. Rev. B: Condens. Matter Mater. Phys. 2004, 70, 115214.

(73) Kresse, G.; Hafner, J. Phys. Rev. B: Condens. Matter Mater. Phys. 1993, 47, 558-561.

(74) Kresse, G.; Hafner, J. J. Phys.: Condens. Matter 1994, 6, 82458257.

(75) Kresse, G.; Furthmüller, J. Comput. Mater. Sci. 1996, 6, 15-50.

(76) Kresse, G.; Furthmüller, J. Phys. Rev. B: Condens. Matter Mater.

Phys. 1996, 54, 11169.

(77) Krukau, A. V.; Vydrov, O. A.; Izmaylov, A. F.; Scuseria, G. E. J. Chem. Phys. 2006, 125, 224106.

(78) Walsh, A.; Da Silva, J. L.; Wei, S.-H.; Körber, C.; Klein, A.; Piper, L.; DeMasi, A.; Smith, K. E.; Panaccione, G.; Torelli, P.; et al. Phys. Rev. Lett. 2008, 100, 167402.

(79) Kehoe, A. B.; Scanlon, D. O.; Watson, G. W. Phys. Rev. B: Condens. Matter Mater. Phys. 2011, 83, 233202.

(80) Godinho, K. G.; Carey, J. J.; Morgan, B. J.; Scanlon, D. O.; Watson, G. W. J. Mater. Chem. 2010, 20, 1086-1096.

(81) Xiao, Z.; Lei, H.; Zhang, X.; Zhou, Y.; Hosono, H.; Kamiya, T. Bull. Chem. Soc. Jpn. 2015, 88, 1250-1255.

(82) Dronskowski, R.; Bloechl, P. E. J. Phys. Chem. 1993, 97, 86178624

(83) Hao, F.; Stoumpos, C. C.; Cao, D. H.; Chang, R. P.; Kanatzidis, M. G. Nat. Photonics 2014, 8, 489-494.
(84) Qiu, J.; Qiu, Y.; Yan, K.; Zhong, M.; Mu, C.; Yan, H.; Yang, S. Nanoscale 2013, 5, 3245-3248.

(85) van der Marck, S. C. Phys. Rev. E: Stat. Phys., Plasmas, Fluids, Relat. Interdiscip. Top. 1997, 55, 1514-1517.

(86) Umari, P.; Mosconi, E.; De Angelis, F. Sci. Rep. 2014, 4, 4467.

(87) Persson, C.; Zhao, Y.-J.; Lany, S.; Zunger, A. Phys. Rev. B: Condens. Matter Mater. Phys. 2005, 72, 035211.

(88) Scanlon, D. O. Phys. Rev. B: Condens. Matter Mater. Phys. 2013 87,161201

(89) Freysoldt, C.; Grabowski, B.; Hickel, T.; Neugebauer, J.; Kresse, G.; Janotti, A.; Van de Walle, C. G. Rev. Mod. Phys. 2014, 86, 253-305.

(90) Gordy, W.; Thomas, W. O. J. Chem. Phys. 1956, 24, 439-444.

(91) Ghosez, P.; Gonze, X.; Lambin, P.; Michenaud, J.-P. Phys. Rev. B: Condens. Matter Mater. Phys. 1995, 51, 6765.

(92) Larson, A. C.; Von Dreele, R. B. General Structure Analysis System; MS-H805; LANSCE: Los Alamos, NM, 1994.

(93) Toby, B. H. J. Appl. Crystallogr. 2001, 34, 210-213.

(94) Wang, J.; Toby, B. H.; Lee, P. L.; Ribaud, L.; Antao, S. M.; Kurtz, C.; Ramanathan, M.; Von Dreele, R. B.; Beno, M. A. Rev. Sci. Instrum. 2008, 79, 085105.

(95) Hammersley, A.; Svensson, S.; Hanfland, M.; Fitch, A.; Hausermann, D. High Pressure Res. 1996, 14, 235-248.

(96) Juhas, P.; Davis, T.; Farrow, C. L.; Billinge, S. J. J. Appl. Crystallogr. 2013, 46, 560-566.

(97) Farrow, C.; Juhas, P.; Liu, J.; Bryndin, D.; Božin, E.; Bloch, J.; Proffen, T.; Billinge, S. J. Phys.: Condens. Matter 2007, 19, 335219.

(98) Momma, K.; Izumi, F. J. Appl. Crystallogr. 2011, 44, 1272-1276.

(99) Zhang, X.; Manno, M.; Baruth, A.; Johnson, M.; Aydil, E. S.; Leighton, C. ACS Nano 2013, 7, 2781-2789.

(100) Perkins, C. L.; Hasoon, F. S. J. Vac. Sci. Technol., A 2006, 24, 497-504.

(101) Kraut, E.; Grant, R.; Waldrop, J.; Kowalczyk, S. Phys. Rev. B: Condens. Matter Mater. Phys. 1983, 28, 1965.

(102) Chambers, S. A.; Droubay, T.; Kaspar, T. C.; Gutowski, M.; Van Schilfgaarde, M. Surf. Sci. 2004, 554, 81-89.

(103) Kresse, G.; Joubert, D. Phys. Rev. B: Condens. Matter Mater. Phys. 1999, 59, 1758-1775.

(104) Perdew, J. P.; Burke, K.; Ernzerhof, M. Phys. Rev. Lett. 1996, 77, 3865-3868

(105) Chen, S.; Gong, X.; Walsh, A.; Wei, S.-H. Appl. Phys. Lett. 2009, 94, 041903.

(106) Ganose, A.; Savory, C. N.; Scanlon, D. O. J. Phys. Chem. Lett. 2015, 6, 4594-4598.

(107) Scanlon, D. O.; Walsh, A. Appl. Phys. Lett. 2012, 100, 251911.

(108) Yuan, Y.; Xu, R.; Xu, H.-T.; Hong, F.; Xu, F.; Wang, L.-J. Chin. Phys. B 2015, 24, 116302.

(109) https://github.com/SMTG-UCL/vacancy-double-perovskites.

(110) Zunger, A.; Wei, S.-H.; Ferreira, L.; Bernard, J. E. Phys. Rev. Lett. 1990, 65, 353.

(111) Romero, A.; Cardona, M.; Kremer, R.; Lauck, R.; Siegle, G.; Serrano, J.; Gonze, X. Phys. Rev. B: Condens. Matter Mater. Phys. 2008, $78,224302$.

(112) Skelton, J. M.; Parker, S. C.; Togo, A.; Tanaka, I.; Walsh, A. Phys. Rev. B: Condens. Matter Mater. Phys. 2014, 89, 205203.

(113) Togo, A.; Oba, F.; Tanaka, I. Phys. Rev. B: Condens. Matter Mater. Phys. 2008, 78, 134106.

(114) Maintz, S.; Deringer, V. L.; Tchougréeff, A. L.; Dronskowski, R. J. Comput. Chem. 2013, 34, 2557-2567.

(115) Paier, J.; Marsman, M.; Kresse, G. Phys. Rev. B: Condens. Matter Mater. Phys. 2008, 78, 121201.

(116) Yang, J.; Siempelkamp, B. D.; Liu, D.; Kelly, T. L. ACS Nano 2015, 9, 1955-1963.

(117) Walsh, A.; Butler, K. T. Acc. Chem. Res. 2014, 47, 364-372.

(118) Ganose, A. M.; Butler, K. T.; Walsh, A.; Scanlon, D. O. J. Mater. Chem. A 2016, 4, 2060-2068.

(119) Henkelman, G.; Arnaldsson, A.; Jónsson, H. Comput. Mater. Sci. 2006, 36, 354-360.

(120) Tang, W.; Sanville, E.; Henkelman, G. J. Phys.: Condens. Matter 2009, 21, 084204. 
(121) Hacene, M.; Anciaux-Sedrakian, A.; Rozanska, X.; Klahr, D.; Guignon, T.; Fleurat-Lessard, P. J. Comput. Chem. 2012, 33, 25812589.

(122) Hutchinson, M.; Widom, M. Comput. Phys. Commun. 2012, $183,1422-1426$. 